\title{
Damaris Nübling
}

\section{Von Elisabeth zu Lilly, von Klaus zu Nico: Zur Androgynisierung und Infantilisierung der Rufnamen von 1945 bis 2008}

\section{Anlass und Zielsetzung}

Im Jahr 2003 erschienen erstmals zwei Arbeiten, die sich mit Rufnamenstrukturen und Geschlechtsklassifikation im Deutschen befassen: Erstens „Naming Gender - Empirische Untersuchungen zur phonologischen Struktur von Vornamen im Deutschen" von Susanne Oelkers, wo deutsche Rufnamen aus den 1990er Jahren auf ihre phonologischen Strukturen hin untersucht werden und die Frage verfolgt wird, inwieweit damit auch Weiblichkeit und Männlichkeit kodiert wird (s. auch Oelkers 2004). Dass die deutschen Rufnamen gesetzlich zu sog. Geschlechtsoffenkundigkeit verpflichtet sind, ist bekannt, doch wurde nie untersucht, worin oder worauf genau Geschlecht markiert bzw. bezogen wird. Was also macht eine Doris weiblich und einen Boris männlich - bzw. eine Janina weiblicher und einen Horst männlicher? Auch wurde nie ernsthaft hinterfragt, ob eine onymische Geschlechtsspezifikation überhaupt existieren muss (vgl. dazu die gegenwärtige Diskussion um die radikale Aufhebung der sexusspezifischen Rufnameninventare in Schweden, initiiert durch die feministische Partei „Feministiskt Initiativ“"1). Damit transportieren Rufnamen, obwohl dies strenggenommen gegen die Eigennamendefinition spricht, eine semantische Information: Sexus.

Zweitens erschien im gleichen Jahr „Die Moderne und ihre Vornamen“ des Soziologen Jürgen Gerhards. Für uns ist Kapitel 8 „Geschlechtsklassifikation durch Vornamen und Geschlechtsrollen im Wandel“ von Interesse.2 Ziel dieses Bandes ist es, „anhand der Vergabe von

1 Hier die entsprechende Passage aus dem Partciprogramm: „Fi I= Feministiskt Initiativ] ska verka för att namnlagen ändras sẳ att alla människor har rätt att ta förnamn utan att det strrs ar biologiskt kön. Denna rätt ska även gälla för föräldrar när de namnger sina barn." strrs ar biologiskt kön. Denna rätt ska även gälla fơr föräldrar när de namnger sina barn."
- Übersetzung (DN): ,Feministiskt Initiativ wird sich dafür einsetzen, dass das Namengesetz geändert wird, so dass alle Menschen das Recht haben, Vornamen anzunehmen, ohne dass dies durch das biologische Geschlecht gesteuert wird. Dieses Recht soll auch für E1tern gelten, wenn sie ihre Kinder benennen" (http:/ www.feministisktinitiativ.se/for_en_ feministisk_politik. php, Nr. 91).

2 Ebenfalls im Jahr 2003 erschien von Gerhards der gleichnamige Beitrag ,Geschlechtsklassifikation durch Vornamen und Geschlechtsrollen im Wandel" (2003b), der Kapitel 8 seines Buches (2003a) entspricht. 
Vornamen kulturelle MLdernisierungsprozesse für die letzten einhundert Jahre sowohl empirisch zu beschreiben als auch durch Rekurs auf strukturelle Veränderungen zu erklären“ (29). Da die Untersuchung von Oelkers (2003) nur sunchron angelegt ist, erhofft man sich von dem Kapitel zur Geschlechtsklassifikation ron Gerhards (2003a) Aufschluss über die Diachronie der onymischen Markierung von Geschlecht. Doch erweist sich hier, dass die Analrse aus linguistischer Perspektive zu kurz greift, da nur der Namenauslaut betrachtet wird.

Das Hauptziel dieses Beitrags besteht darin, anhand einer tiefergehenden prosodisch-phonologischen Analyse der häufigsten Rufnamen von 1945-2008 der Frage nachzugehen, ob im Iaufe der Zeit eine Androgrnisierung unserer Rufnamen dahingehend stattgefunden hat, dass Strukturen, die bislang dominant für das eine Geschlecht galten, zunehmend auch für das andere Geschlecht gewählt werden bzw. geschlechtsauch fur das andere Geschlecht gewahl werden bzw 3 Fin weiteres präferente Strukturen nivelliert oder gar abgebaut werden. ${ }^{3}$ Ein weitere Ziel besteht darin, auf onymischer Ebene der These nachzugehen, dass in verschiedenen gesellschaftlichen Bereichen eine sog. Informalisierung und Intimisierung stattgefunden habe, die sich möglicherweise in heutigen Namen wie Lilly oder Nico statt früher Elisabeth und Nikolans niederschlagen. ${ }^{4}$

\section{Androgynisierung}

\subsection{Bisherige Forschungen zu Rufname und Geschlecht}

Die bereits erwähnte Untersuchung von Gerhards (2003a, b) basiert auf Geburtseinträgen von 1894-1994 bzw. 1998 in der westdeutschen Klein stadt Gerolstein und der ostdeutschen Stadt Grimma. Gerolstein ist mehrheitlich katholisch, Grimma evangelisch bzw. seit 1949 eher konfessionslos. In Gerolstein wurden die jeweils ersten 100 Geburten von 1894 1950 in vierjährigen, von 1950-1994 in zweijährigen Abständen erfasst. Ähnlich in Grimma: Hier wurden die jeweils ersten 100 Geburten von 1894-1998 in zweijährigen Abständen ermittelt, d.h. jedem erhobenen Jahr liegen ca. 50 Mädchen- und 50 Jungennamen zugrunde (darin sind Mehrfachvergaben eines Namens enthalten). Den Geburtseinträgen

3 Zu diesem Thema habe ich zwei Aufsätze verfasst, auf denen det erste Teil des vorliegenden Beitrags stark basiert: Eine ausführlichere Version (Nübling 2009a) in „Beitrage 2ur Namenforschung" und eine Kurzfassung (Nübling 2009b) in "Der Deutschunterricht"

4 Verzichtet wird hier auf den weitergehenden Bereich der Phonosemantik (bzw. Psychophonetik), d.h. ob es ,weiblich" bzw. „männlich“ klingende Laute bzw. Klangassoziationen Pribt. Hierfür sei auf Oelkers (2003: 69-123) rerwiesen. wurden einige Daten entnommen, wie das Geburtsdatum, das Geschlecht des Kindes, weitere eventuelle Vornamen, die Religionszugehörigkeit und der Beruf von Mutter und Vater. Diese Informationen dienen vor allem für die Fragestellungen der anderen Kapitel, die sich primär auf Säkularisierungsprozesse, auf den Bedeutungsverlust verwandtschaftlicher Traditionsbindungen, Individualisierungsprozesse, die Transnationalisierung der Vornamen etc. beziehen. Nur das Kapitel „Geschlechtsklassifikation durch Vornamen und Geschlechtsrollen im Wandel" ist für uns von Interesse. Hierfür berücksichtigt Gerhards nur die Jahre 1950-1990, da der für die Geschlechtsrollen wichtigste Wandel, die Gleichberechtigung und Gleichstellung der Frau, im Gefolge von 1968 eingeleitet wurde. Dies betrifft nicht nur die Rollenerwartungen an Frau und Mann, sondern auch die faktische Geschlechterrollendifferenz, ersichtlich etwa an der Kleidung, der Anzeigenwerbung, vermehrter Geschlechterrepräsentanz in der Sprache und anderen sozialen Veränderungen. Zwei Fragen leiten den Autor:

In welchem Maße wird über die Benutzung von Vornamen das Geschlecht von Personen Hassifiziert und lässt sich diesbezüglich ein sozialer Wandel der $\Delta \mathrm{b}$ nahme der Geschlechtseindeutigkeit von Vornamen feststellen? Greifen die Eltern je nach Geschlecht des Kindes auf unterschiedliche Namenskulturkreise zurück, sind damit geschlechtsspezifische Rollenvorstellungen verbunden und haben sich diese im Zeitverlauf der letzten 100 Jahre verändert? (152)

Seine Hypothese lautet, dass der gesellschaftlichen Annäherung der Geschlechterrollen eine Androgynisierung der Rufnamen folgen sollte.

Ob ein Rufname ein Mädchen- oder ein Jungenname ist, kann auf Konvention und damit purem Erfahrungswissen beruhen (vgl. Doris Boris) - ähnlich der Tatsache, dass wir jedem Substantiv ein grammatisches Geschlecht zuordnen können, obwohl sich dieses nur sehr bedingt aus seiner phonologischen Struktur (oder anderen Merkmalen) ergibt. Geschlecht könnte also als Lexikoneintrag zum Namen mitgelernt werden. Dem ist jedoch faktisch nicht immer so. Man erschließt, gerade bei unbekannten Rufnamen, das Geschlecht des Trägers/der Trägerin ,irgendwie“ aus der phonologischen Struktur. Dabei sind overte Verfahren wie Movierungen vom Typ Martin - Martina eher selten (die umgekehrte Ableitungsrichtung existiert im Deutschen nicht).

Dass ein phonetisches Wissen besteht, legen Tests aus den USA nahe, die Gerhards resümiert. In den USA dürfen Rufnamen frei kreiert werden, und es besteht auch keine Verpflichtung zur Geschlechtsoffenkundigkeit. Allerdings hat das Experiment von Lieberson/Mikelson (1995) gezeigt, dass bei solchen erfundenen Namen dennoch feste Geschlechtszuordnungen vorgenommen werden: Einer Zufallsauswahl von 16 selbst kreierten (und auch wirklich vergebenen) Neunamen mussten 225 Amerikane- 
rInnen das ihres Erachtens „richtige“ Geschlecht zuordnen. Das Gleiche wiederholte Gerhards mit 184 Leipziger Studierenden. In beiden Ländern wurden diese 16 Namen in erstaunlich übereinstimmender Weise sexusklassifiziert (gleich ob das Kind nun tatsächlich ein Mädchen oder ein Junge war): Zu (teilweise weit über) zwei Dritteln wurde Lamecca, Timitra, Maleka, Sukoya, Furelle, Shatrye weiblich und Husan, Oukayod, Cagdas, Gerais, Rasbueen männlich klassifiziert - was auch den tatsächlich benannten Geschlechtern entsprach, abgesehen von Furelle, das einen Mann bezeichnete. Probleme bereiteten die Namen Shameki $(q)$, Chanti $(\delta)$, Kariffe $(q)$ und Trician $(9)$. Bei letzterem wurde das Geschlecht zwar in den USA zu fast $70 \%$ richtig erschlossen, in Deutschland aber nur zu $7 \%$. Bei Jorell $\left(\delta^{\dagger}\right)$ tippten in den USA tast $80 \%$ richtig, in Deutschland $50 \%$. Da da Erfahrungswissen bei solchen neuen Namen nicht greifen kann, müssen es phonologische Strukturen sein. Der Auslaut $-a$, auch der auf - - , löst offensichtlich eine weibliche Klassifikation aus, Namen auf Konsonant eine männliche, bei solchen auf $-i$ ergeben sich Probleme. Gerhards schließt daraus:

Zur Bestimmung einer typisch männlichen und typisch weiblichen Phonetik kann Zur Be man sich auf die Enlaure kenemen auf das Gedann, so die Vermutung, schließt man von
schlecht des Namens (Gerhards 2003a: 159). Allerdings scheint Gerhards nicht von der phonologischen (oder gar phonetischen), sondern nur von der graphematischen Oberfläche auszugehen, auch wenn von „fonetischen Analysen“ (Gerhards 2003b) die Rede ist 5 Gerhards fasst alle Namen zwischen 1950 und 1998, die auf $-a$ oder - $e$ enden, zu den weiblichen und die auf $-n,-s,-d$ und $-r$ endenden $z u$ den männlichen Rufnamen, wohl wissend, dass es gegenläufige Namenstrukturen gibt wie Sascha $(\hat{j})$ und Doris (q). Anschließend überprüft er, ob sich die Geschlechtsklassifikation über diese Namenausgänge im Zeitverlau verändert, d.h. ob immer mehr konsonantisch auslautende Mädchennamen bzw immer mehr auf - a oder -e auslautende Jungennamen gewählt werden. Das Eroebnis ist negativ, der Auslaut bleibt im Zeitverlauf stabil. Die onymische Markierung von Geschlecht scheint sich also nicht zu ändern, eine Androgynisierung bleibt aus, die gesellschaftlichen Umbrüche schlagen sich nicht in den Namen nieder:

Das Errebnis unserer Analysen ist damit relativ eindeutig: Vermännlichungs- un Verweiblichungsprozesse von Vornamen lassen sich für die Zeit von 1950 bis

So ist zu vermuten, dass die Namen Simone, Yronne, Andre und Marie bei Gerhards alle auf

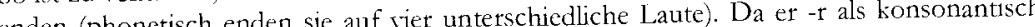
enden (phonetsch (faktisch ist es der Vokal [e]), ist zu vermuten, dass er ausschließlich Grapheme gezählt hat.
1990 nicht nachzeichnen. Die Klassifikation des Geschlechts eines Kindes durch den Vornamen hat sich im Zeitverlauf nicht verändert (Gerhards 2003a: 164).

Die Klassifikation von Menschen nach ihrem natürlichen Geschlecht stelle offensichtlich ,einen so fundamentalen Mechanismus der Ordnungsbildung“ dar, „dass dieser indifferent ist gegenüber dem Wandel der Geschlechtsrollen [...]" (165).

Dagegen sieht Gerhards in der kulturellen Herkunft eines Rufnamens eine Kodierungsmöglichkeit von Gender als konstruiertem sozialem Geschlecht. Dazu unterteilt er seine Rufnamen in christliche und deutsche (= germanische). Die untersuchten 100 Jahre zeigen, dass der Anteil der deutschen Namen bei den Mädchen schon immer gering war (um die 20\%) und 1994 gegen Null geht, während er bei den Jungen immer höher war (1894 betrug er ca. 50\%), ab 1942 auf über 60\% ansteigt und seit 1964 zurückgeht, um heute ähnlich niedrig zu liegen wie bei den Mädchen. Umgekehrt ist der Anteil christlicher Namen bei den Mädchen bis 1964 höher als bei den Jungen. Daraus folgert Gerhards (2003a), dass man Jungen „mit einer deutsch-nationalen, aktiv gestaltenden Öffentlichkeitsrollec assoziiere, die Mädchen dagegen ,mit einer übersinnlichen, außerweltlichen Sphäre“ (169). Frauen ,bleiben stärker christlich religiös verhaftet, ihre Sphäre liegt im Privaten und im Übersinnlichen" (171). Auch dass Mädchennamen im Laufe der Zeit häufiger wechseln als Jungennamen, wird wie folgt interpretiert: Jungen werden eher auf das Tradionelle, Feste, Stabile verpflichtet, Mädchen gewähre man ,größere Offenheit und Leichtigkeit“ (167). Hier also finde die Befrachtung der Rufnamen mit geschlechtstypischen Rollenvorstellungen (Gender) statt. Dagegen praktiziere man auf der Ebene der Namenphonetik, genauer: des Auslauts, die („natürliche“) Geschlechtsklassifikation als Sexusanzeige, die sich auch kaum verändert habe und ,indifferent ist gegenüber sozialem Wandel" (173). Dies sei insgesamt „ein konsistentes, wenn auch zum Teil unerwartetes Ergebnis" (172)

Aus linguistisch-onomastischer Perspektive ist dem mehrerlei entgegenzuhalten: Spätestens seit dem 2. Weltkrieg (für die Zeit davor gibt es keine Befragungen) folgt die Rufnamenvergabe primär euphonischen Kriterien: Elternbefragungen in den $1960 \mathrm{er}$ bis $1980 \mathrm{er}$ Jahren sprechen alle für die Dominanz der Euphonie, wozu man auch (manchmal getrennt aufgeführte) Kriterien wie „Kürze“, „Schlichtheit", „Seltenheit" und „Harmonie mit dem Familiennamen“ zu zählen hat. Als nicht euphonisch motivierte Vergabekriterien sind allenfalls noch die Nachbenennung nach Familie, Patenschaft, Heiligen oder nach anderen konkreten Vorbildern zu finden, aber nicht Herkunft oder Etymologie des Namens (s. Debus 1985, 1987). Außerdem dürfte den wenigsten Eltern bekannt sein (auch nicht unbewusst), dass so ähnlich strukturierte Namen wie Peter und Hans 
christlich sein sollen, Dieter und Heinzaber deutsch. Ähnliches gilt für Rita (christlich), Britta (keltisch) sowie Helga (germanisch).

Außerdem wird man der Kodierung von Weiblichkeit und Männlichkeit kaum gerecht, indem man hierfür nur den Namenauslaut heranzieht. Zumindest für das Deutsche ist dieses Vorgehen unzureichend. Hierfür muss der gesamte Wortkörper in Betracht gezogen werden. Oelkers (2003) weist nach, dass im Deutschen Geschlecht (ob Sexus oder eher Gender, muss hier offen bleiben) auf dem gesamten Namen kodiert wird. Grundsätzlich gibt es nach Oelkers (2003: 41 ff.) drei Möglichkeiten, Geschlecht onymisch zu markieren:

a) semantisch

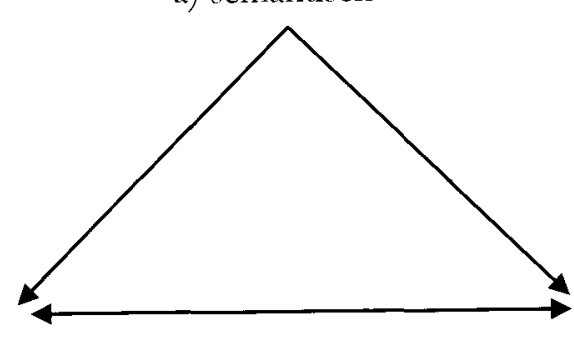

b) formal

c) konventionell

Abb 1. Formen der Geschlechtsmarkierung an Personennamen

a) Beim semantischen Prinzip muss der Name inhaltlich verständlich, also sprechend sein. Einige Kulturen, in Zügen auch das Germanische mit seinen sog. programmatischen Rufnamen, praktizieren dieses Prinzip, z.B. das Chinesische, das Japanische, das Türkische. Hierbei sind sozialen Geschlechtszuschreibungen (Genderungen) Tür und Tor geöffnet, je nachdem, welche Attribute oder erstrebenswerte Eigenschaften man den Geschlechtern jeweils zuweist und wie stark sich diese überschneiden bzw. ausschließen. Oelkers (2003: 41) erwähnt für das Türkische die Männernamen Yzlmaそ, furchtlos' und Kemal Reife' sowic die Frauennamen Aynur ,Mondlicht', Gül ,Rose und Inci ,Perle'.

b) Beim formalen Prinzip kommen Affixe oder andere eindeutige, segmentierbare Namenbestandteile zum Einsatz, etwa Movierungssuffixe wie in nhd. Martina, Bernhardine. Christiane. Dieses Verfahren ist im Deutschen nur schwach ausgeprägt. Viel eher ist das Italienische diesem Typus zuzuordnen, da aufgrund spezifischer Namenauslaute die Geschlechter tatsächlich (fast) eindeutig erschließbar sind: Wie Bardesono (2008) ermittelt hat, lauten 95\% der weiblichen Rufnamen auf $-a$ und $4 \%$ auf $-e$ aus, während $77 \%$ der männlichen Rufnamen auf
$-0,14 \%$ auf $-e$ und $4 \%$ auf $-a$ enden (sowie $3 \%$ auf $-i$ und $1 \%$ auf $-l$ ). Dies resultiert aus dem formalen System des Lateinischen, vgl. Claudia vs. Claudl-us.

c) Konventionelle Systeme sehen zwei getrennte Inventare an Frauenund Männerrufnamen vor, die man im Extremfall auswendig lernen muss. Hierzu ist das Deutsche zu rechnen, auch wenn, wie Oelkers gezeigt hat, das phonologische Material der Namen stark zur Geschlechtskennzeichnung verwandt wird und es erlaubt, auch neue Namen nach Sexus zu klassifizieren. Die Prinzipien sind jedoch subtiler, d.h. weniger overt wie beim formalen Prinzip und wie von Gerhards (2003a, b) angenommen, der mit seiner Fokussierung einzig des Auslauts (bzw. Auslautgraphems) dem Deutschen ein formales System unterstellt.

Was die (bis dato kaum untersuchten) diachronen Übergänge zwischen diesen drei Prinzipien der Geschlechtsmarkierung betrifft, so deuten die Pfeile an, welche Pfade die wichtigsten sind.

Geschlechtsambige Namenkulturen sind insgesamt selten (zu einem Überblick hierzu s. Brylla 2001b). Doch erlauben manche Länder wie z.B. die USA den Gebrauch geschlechtsneutraler Namen. Dass AmerikanerInnen dennoch, selbst wenn sie Namen erfinden, von geschlechtstypischen Mustern Gebrauch machen, wurde oben im Zusammenhang mit der Studie von Lieberson/Mikelson (1995) bereits erwähnt. Barry/Harper $(1982,1993)$ haben sog. unisex names in den USA untersucht und sind zu dem Ergebnis gelangt, dass diese nur während einer vergleichsweise kurzen Zeit wirklich für beide Geschlechter verwendet werden. Im Laufe der Zeit werden sie immer öfter exklusiv an Mädchen vergeben - und es hat sich herausgestellt, dass unisex names mehrheitlich einstigen Männernamen entstammen:

This prediction [dass unisex names öfter männlichen Ursprungs sind und sich zu weiblichen weiterentwickeln als umgekehrt] is based on cultural attitudes, males being favored but more limited by sex stereotyping. Therefore, parents are more likely to give their daughter a traditional male name than to give their son a traditional female name. Lnisex names are avoided for a son but not for a daughter (Barry/Harper 1982: 15).

In der Geschichte des Deutschen gab es nie geschlechtsambige Rufnamen in größerem Umfang (es handelt sich um wenige und wohl deshalb ständig diskutierte Einzelfälle vom Schlage Eike, Kim, Andrea). Hier hat insgesamt ein Wandel von System (a) und (b) > (c) stattgefunden (mit Anteilen an (b); s. hierzu Oelkers 2003: 45-56). Semantische Anteile des (germanischen) Systems (a) kamen wenn, dann in Adjektiven und Tierbezeichnungen zum Ausdruck. Insgesamt, dies wird immer wieder betont, haben die 
Namen beider Geschlechter an den Bereichen Kampf, Ehre und Heldentum gleichermaßen teil (s. Andersson 1998, Brylla 2001a, b). Vorrangig hat jedoch das Genus des substantivischen Zweitglieds (der germanischen Namenkomposita) die Geschlechtsspezifikation geleistet. Dies hat als formales Verfahren (b) zu gelten. Nebenbei: Es ist erstaunlich, dass bei der langen und nicht nur in der Genderlinguistik geführten Diskussion um - Verhältnis zwischen Genus und Sexus diese enge und ausnahmslos geltende Genus/Sexus-Verschränkung nie gesehen wurde. ausnahmslos geltende Genus/Ser substantivischen Namenzweitglieder war Der semantische Gehär für die Sexuszuweisung war die reine Genuszusogar sekun

Seit dem Mittelalter gilt für das Deutsche das konventionelle Prinzip, doch bilden sich heute im Zuge der freien Namenvergabe, die man frühestens ab dem 19. Jh. ansetzt, immer mehr euphonisch motivierte

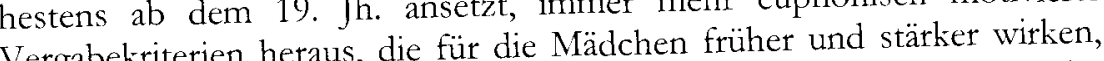
für die Jungen später und schwächer (Debus 1985). Von juristischer Seite wird seit jeher Geschlechtsoffenkundigkeit gefordert, wie immer diese bestimmbar sein soll. Diese Geschlechtskennzeichnung erfolgt über bestimmbar sein soll. Diese Gëcher verankerte phonologische Struk-

subtile, auf dem gesamten Wortkörper verankerte phonologische
turmuster, denen im Folgenden genauer nachzugehen sein wird.

\subsection{Die 20 häufigsten Rufnamen seit 1945 und ihre Aufbereitung für die Untersuchung}

Da hier nicht der Raum ist, alle Top-20-Rufnamen seit 1945 aufzulisten, beschränke ich mich darauf, die Spitzenreiter von 1945, 1975 und 2005 aufzulisten.

Schon ein erster Blick auf Tabelle 1 erweist, dass es tiefgreifende Veränderungen in der Rufnamenstruktur gegeben hat. Nicht nur hat sich der Namenbestand jeweils komplett erneuert, es haben sich auch die prosodisch-phonologischen Strukturen statk verändert 2005 scheinen die Namen tenderiell kürzer zu sein, vor allem weicher", sonorer, doch Namen tendenziell kürzer zu sein, ve lassen sich Details noch kaum ablesen, auch nicht schlechterdifferente Entwicklungen hindeuten könnten. Eine solche Analyse soll in Abschnitt 2.3 vorgenommen werden.

\begin{tabular}{|l|l|l|l|l|l|l|}
\hline \multirow{nyyyyyy}{*}{} & \multicolumn{2}{|c|}{1945} & \multicolumn{2}{c|}{1975} & \multicolumn{2}{c|}{2005} \\
\hline 1 & Reiblich & männlich & weiblich & männlich & weiblich & männlich \\
\hline 2 & Monika & Peter & Stefanie & Markus & Hanna & Leon \\
\hline 3 & Karin & Klaus & Nicole & Nichael & Anna & Luka \\
\hline 4 & Ursula & Wolfgang & Kathrin & Stefan & Lea(h) & Finn \\
\hline 5 & Brigitte & Jürgen & Tanja & Andreas & Lena & Niklas \\
\hline 6 & Bärbel & Uwe & Anja & Thomas & Laura & Jonas \\
\hline 7 & Elke & Bernd & Yvonne & Alexander & Emilie & Tim \\
\hline 8 & Ingrid & Karl & Julia & Sven & Lara & L(o)uis \\
\hline 9 & Helga & Horst & Claudia & Thorsten & Sophie & Jan \\
\hline 10 & Christa & Dieter & Melanie & Jan & Marie & Paul \\
\hline 11 & Gisela & Günther & Katja & Matthias & Julia & Felix \\
\hline 12 & Hannelore & Heinz & Nadine & Frank & Sarah & Jannick \\
\hline 13 & Jutta & Rainer & Silke & Martin & Lilli & Julian \\
\hline 14 & Barbara & Nichael & Andrea & Jens & Emma & Max \\
\hline 15 & Heike & Manfred & Sonja & Sebastian & Lina & Philipp \\
\hline 16 & Christel & Rolf & Susanne & Marco & Johanna & Maximilian \\
\hline 17 & Marion & Gerhard & Bettina & Oliver & Ne(e)le & Ben \\
\hline 18 & Erika & Werner & Daniela & Andre/é & Alina & Moritz \\
\hline 19 & Angelika & Gerd & Sabine & Mark & Luisa & Nico \\
\hline 20 & Anke & Helmut & Alexandra & Daniel & Sophia & Tom \\
\hline
\end{tabular}

Tab. 1: Die 20 häufigsten Rufnamen im Jahr 1945, 1975 und $2005^{\circ}$

Um der zentralen Frage nachzugehen, auf welche Namenstrukturen Eltern präferent zugreifen, interessieren vor allem die faktisch vergebenen Rufnamen (Tokens) und nicht etwa das Gesamtinventar an möglichen Rufnamen (Types). Bekanntlich divergieren Types und Tokens oft erheblich, d.h. Eltern nutzen nur einen kleinen Ausschnitt der Namenpools. Dies begründet, weshalb ich mich im Folgenden auf die jeweils 20 häufigsten Mädchen- und Jungennamen beziehe.

In der Literatur wird wenn, dann meist nur auf die Top 10 Bezug genommen, doch erscheint mir dies als zu begrenzt, zu wenig aussagekräftig

6 Von Schreibvarianten wird hier weitestgehend abgesehen, da nur die phonologische Seite interessiert. Zu den Schreibungen s. die Listen in „beliebte-vornamen“. Aus Gründen der Lesbarkeit wird hier nur dann transkribiert, wenn es auf Details ankommt. Allen Auswertungen liegen selbstverständlich Transkriptionen zugrunde. 
und als zu sehr von möglicherweise anderweitig bedingten Modephäno menen abhängig zu sein. Bei den hier zugrunde gelegten Top 20 sind die einzelnen Rangpositionen für uns uninteressant: Es soll nur darum gehen, die häufigsten Namen eines Jahrgangs zu erfassen. Frühere Schätzungen bzw, regional begrenzte Untersuchungen gehen davon aus, dass die häufiosten Namen von jeweils ca. 3-6\% der Neugeborenen getragen wurden (so z.B. Debus 1977 für Kiel). Dabei gehen die Prozentzahlen mit wurden (so z.B. Debus der Zeit stark zurück, d.h. die Namengebung wird individueller, etwas mehr bei den Mädchen als bei den Jungen (Debus 1976b). Exakte, bundesweit gültige Zahlen sind nicht verfügbar. Für vier Städte (Westberlin, Wiesbaden, Heidelberg, Tübingen) legt Seibicke (1991: 110) einige Zahlen für das Fnde der 1980er Jahre vor: Die jeweiligen Spitzenreiter bewegen . $6,2 \%$ und die Werte für den 1.-10. sich tatsachlich zwischen $3,6 \%$ und $6,2 \%$, und Namen, also die Top 10, reichen von 24\% (Berlin, Jungen) bis $42,2 \%$ (Tübingen, Jungen). Auch wieviel Prozent der Kinder einen Namen de Top 20 tragen, wurde ermittelt. Hier variieren die Werte beträchtlich: Sie oszillieren zwischen 25,6\% (Heidelberg. Mädchen) und 67,3\% (Tübingen, Jungen). ,M] it 20 Jungen- und 20 Mädchennamen [gemeint sind die Top 20 - DN] erfaßt man rund die Hälfte aller Erst- und Einzelvornamen", chätzt Seibicke (1991: 112). Heute (2008) ist dieser Wert deutlich geringer: Nur noch 13,41\% der Mädchen und 14,25\% der Jungen tragen nach Ausweis von beliebte-vornamen.de" einen Top-20-Namen.?

Um die Datenmenge von 1945 bis heute handhabbar zu halten, habe ich Fünfjahresschritte gewählt: 1945, 1950, 1955 etc. bis 2005 und zusätzlich 2008. Dies ergibt insgesamt 14 Jahresschnitte.

Valide und das gesamte Bundesgebiet abdeckende offizielle (amtliche) Daten cibt es bis heute nicht. Dies gilt auch für die jährlich in der Presse erscheinenden Rufnamenstatistiken, die von der Gesellschaft für deutsche Sprabe (GfIS) ermittelt werden. Die datenliefernden Standesämter werden Sprache (GfdS) ermittelt werden. Die datenliefernden Standesănter werden zwar von Jahr zu Jahr zahlreicher (2006 waren es 180), doch erschwer dies umgekehrt die Vergleichbarkeit zwischen den Jahrgängen. Da die GfdS immer nur die 10 häufigsten Rufnamen errechnet und dabei - dies betrifft und verzerrt v.a. die Mädchennamenstatistik - die Zweit- und Detifft und verzerte diese wie Erstrufnamen behandelt statt sie

- Tur 7ürich kommr Moser (2009) für die von 1988-2008 vergebenen Rufnamen zu folgenFur Zurich kommt Moser (2009) fur die von 1980 dass die Verteilung der Namenshäufigden Ergebnissen: ,,[E]s darf nicht vergessen geh, die 2008 im Kanton Zürich geboren wurkeiten sehr flach ist. Von den 14.480 Kindern, die 2008 im Kanton Zurich geboren wurden, tragen nut 163 die beiden populärsten Namen, "Sara oder "Leon - das entspricht etwas mehr als einem Prozent des Totals. Rund ein Viertel (26\%) der Kinder erhalten zwar einen der 100 meistverwendeten Namen aber die restlichen drei Viertel tellen sich rund 4.300 verschiedene Namen. Etwa zwei Drittel aller Namen (64\%) werden sogar nur einmal verwendet. [...] Die Hitliste ist also bloss die winzige Spitze eines Eisbergs. Dic überwäldrende Mehrheit der Fltern wählt andere Namen" (2). herauszurechnen, verzerren typische, immer wiederkehrende Zweitrufnamen wie Marie, Maria, Sopbie schon seit Jahren die Top 10, indem sie dauerhaft die ersten Ränge besetzen bzw. blockieren.

Deshalb beziehe ich mich auf die Quelle ,www.beliebtevornamen.de“. Diese Statistik zieht ebenfalls Standesämter heran, zusätzlich Geburtskliniken, Geburtshäuser etc. Der Statistik ron 2008 liegen z.B. genau 109.305 Geburtsmeldungen aus 301 Standesämtern und Geburtseinrichtungen zugrunde, was $16 \%$ aller Neugeborenen entspricht. Hier werden auch jeweils die ersten 250 Ränge ermittelt. Dies ist - neben der guten Zugänglichkeit über das Internet - ein entscheidender Vorteil gegenüber den GfdS-Daten: Man kann sehen, was sich jenseits der Top 10 bzw. Top 20 abspielt. Im Gegensatz zu den Daten der GfdS gehen bei „www.beliebte-vornamen" die Zweit- und Drittrufnamen nicht in die Häufigkeitsstatistik ein, sie werden herausgenommen und extra gezählt. Des Weiteren gehen die Statistiken historisch viel weiter zurück, genau bis 1890. Dies ist für diachrone Untersuchungen von unschätzbarem Wert. Die Daten der GfdS beginnen mit einiger Regelmäßigkeit erst in den 1980er Jahren, frühere Zahlen sind nur für wenige Jahrgänge erhältlich. Um sicher zu gehen, habe ich die Top 10 der GfdS, soweit für die hier interessierenden Jahre überhaupt vorhanden, mit den Top 20 von ,beliebte-vornamen" abgeglichen: Fast ohne Ausnahme sind die Top 10 der GfdS in den Top 20 enthalten, d.h. beide Statistiken konvergieren in höchstem Maß. Eine Ausnahme bildet der typische Zweitrufname Maria, der 1990 - erwartungsgemäß - nur in den Top 10 der GfdS enthalten ist. Die andere Ausnahme bilden David (2000 und 2005) sowie Alexander (2005): Auch Alexander ist ein typischer Zweitvorname und belegt denn auch in der Statistik der häufigsten Zweitvornamen im Jahr 2005 Platz 1 (http://www.beliebte-vornamen.de/2005-zweit-namen.htm). Differenzen gibt es nur in den einzelnen Rangabfolgen, doch diese sind für uns ohne Belang: Es geht hier einzig und allein darum, die häufigsten und damit repräsentativen Rufnamen möglichst vieler Jahrgänge zu ermitteln, gleich ob sie auf Platz 1 oder auf Platz 20 stehen. Im Folgenden werden den Berechnungen die Top 20 von umw. beliebte-vornamen. de zugnunde gelegt.

In einem ersten Schritt wurde das gesamte Rufnamenkorpus transkribiert (20x2x14=560 RufN). Schreibvarianten sind (weitestgehend) unerheblich, sie gingen daher auch nicht in das Korpus ein (es wurde die jeweils üblichere Schreibvariante gewählt). Nur in wenigen Fällen konnte nicht eindeutig transkribiert werden, z.B. bei Andre/André (Akzentposition) oder bei Marcel und Kristin (auch Akzentposition). In solchen Fällen wurden beide Varianten mitgezählt und entsprechend auf 21 statt 20 Namen bezogen. Bei Jessica und Jennifer wurde die engl. Aussprache mit [d] angesetzt. Selbstverständlich müssen die eingetragenen Namenvoll- 
formen zugrundegelegt werden, d.h. dass ein Maximilian Max genann wird, ist zwar naheliegend, aber nicht mit Sicherheit gegeben, zumal Max wird, ist zwar naheliegend, abom 20 vorkommt. Dieses Problem der späteren $\mathrm{Na}$ menverkürzung oder -veränderung gilt für viele andere Namen auch und muss hier außer Betracht bleiben (s. hierzu Abschnitt 3).

Zu phonologischen Details: Die wenigen Affrikaten und Diphthonge wurden

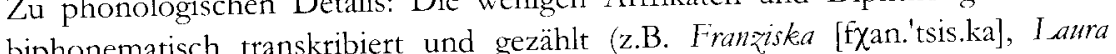
biphonematisch transkribiert und gezählt (z.B. Franziska [fxan.tsis.ka], I aura [lau.ka], Klaus [klaus]). Hiate wurden als zwei Silben gewertet, auch wenn sie bei Allegrosprechweise zu einer Silbe kontrahiert werden konnen (dies ist stark

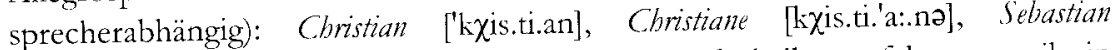
[ze.'bas.ti.an], Julia ['ju:li.a]. So wurde auch deshalb verfahren, weil in vellälen nicht immer davon auszugehen ist, dass tatsächlich vergleichbaren Fallen nicht immer davon *fflo:s.jan], auch Adrian, Marianne kontrahiert wird, vgl. Florian ['flo:.b1.an], kaum *[flo:k.jan], auch Adrian, Marianne etc. Damit ist auch eine Gleichbehandlung mit anderen Hiaten gesichert, dis keinesfalls kontrahiert werden: Andreas [an.'dxe:.as], Michael ['mi.ça.el], Matthias [ma.'ti.as], Marion [ma:.ki.on]. Dieses Vorgehen ist im Fall von [ia] und [ua] durchaus diskutabel; Oelkers (2003) geht hier jeweils von nur einer Silbe aus, d.h. Florian hat zwei und Manuela drei Silben. Da durch die hier vorgenommene prinzipielle Bewertung als Hiate Jungen- wie Mädchennamen gleich behandelt werden, halten sich mögliche Verzerrungen zwischen den Geschlechtern in Grenzen. Auf die Transkription und Berücksichtigung des Knacklauts wurde grundsätzlich verzichtet: Im betonten Vokalanlaut ist er immer anzusetzen, in Fällen wie Micha[el]/Micha[?el] variiert dies regional oder individuell.

Viel wichtiger ist die Berücksichtigung der Phonetik beim deutschen / $\mathrm{r}$-Laut der je nach Position und Umgebung in seiner Realisierung stark schwankt und in keinem Fall als Liquid zu verbuchen ist. Im unbetonten Auslaut nach Schwa is /r/ immer vokalisiert, d.h. hier gilt Peter [pe:te] und Dieter ['di:.te]. Damit lauten (wie dies bei Oelkers 2003 (we dies bei Oelkers 2003 und Gerhards 2003a, b voraussetzen). Das gleiche gilt für die präkonsonantische Position (Bernd ['beent], Werner ['vę.ne]). Im Anlaut und intervokalisch wird /r/ Position (Ben Konsonant als als stimmhafter Frikativ [r] angesetzt (Renate [se.'nar.t], nach Konsonant als

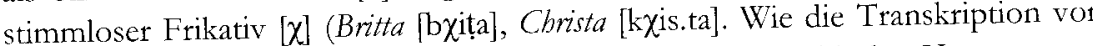
Britta außerdem zeigt, werden (phonetisch reale) ambisibische Konsonanten Britta außerdem zer im Hauptton vorkommen, d.h. die betonten Silben in 'Anna, Bri'gitte oder Mari'anne sind geschlossen. Die Silbengrenze verläuft durch den Briten den daruntergesetzten ambisibischen (Kurz-) Konsonanten, angezeigt durch Punkt. Alle betonten Vokale, denen kein ambisilbischer Konsonant folgt, sind automatisch lang. Dennoch wird die Länge durch [:] markiert. Schließlich wurde auch die Auslautneutralisierung berücksichtigt.
In den wenigen phonologischen Rufnamenanalysen wird m.E. allzu vereinfachend von einer Dichotomie zwischen Vokalen und Konsonanten ausgegangen und deren Anzahl dann oft in Relation (und damit Opposition) zueinander gesetzt. Bekanntlich erstreckt sich zwischen Vokalen und Konsonanten aber ein Kontinuum, das auf der Sonoritätsskala in Abb. 2 abgebildet ist.

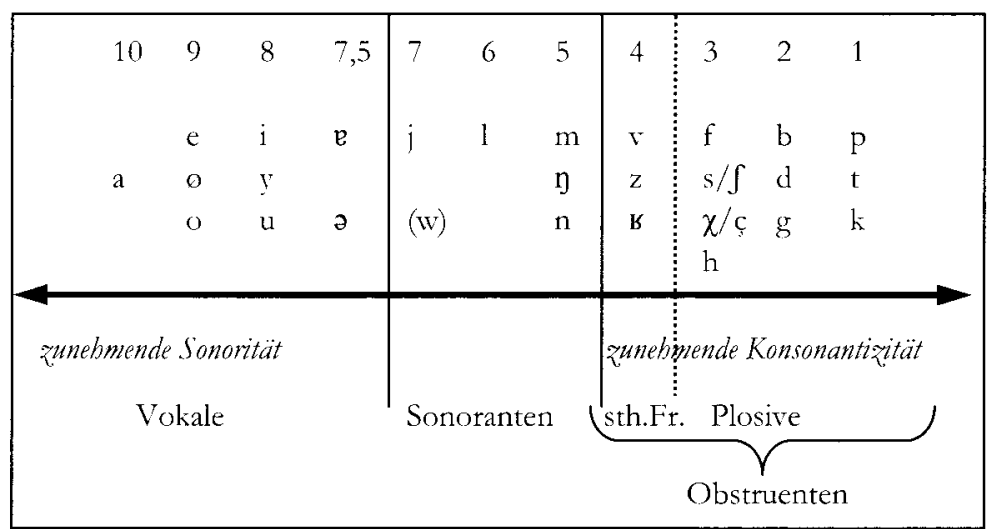

$\Lambda$ bb. 2: Die Sonoritätshierarchie und die Sonoritätswerte

Der linke Senkrechtstrich zeigt die traditionelle Trennung zwischen Vokalen und Konsonanten an. Für unsere Belange besonders wichtig sind die Sonoranten, teilweise auch die stimmhaften Frikative (sth.Fr.), da diese dem Namen einen als weich empfundenen Klang verleihen und heute sehr euphonisch wirken. Nach rechts hin nimmt der Grad an konsonantischer Stärke zu, endend bei den stimmlosen Plosiven. Namen wie Brigitte oder Peter enthalten also starke Sonoritätskontraste, solche wie Mia oder Leon dagegen nur schwache.

Um die Sonorität irgendwie messbar zu machen, wurden Sonoritätswerte vergeben, die sich in der obersten Zeile von Abb. 2 befinden. Dabei soll angenommen werden, dass der Sonoritätsabstand zwischen benach barten Lauten immer 1 beträgt, d.h. die stimmlosen Plosive bekommen den niedrigsten Wert 1, der Vokal / a/ erhält den höchsten Wert 10. Die beiden Schwa-Laute [e] und [ə], die ohnehin nur in unbetonten Silben vorkommen, erhalten als einzige mit 7,5 Punkten einen Zwischenwert.

\subsection{Die Untersuchung und ihre Ergebnisse}

Oelkers (2003: 125-214) hat in ihrer sunchronen Untersuchung, basierend auf einem umfangreichen, repräsentativen Korpus aus den Gesamtdaten (Frequenzminimum: mind. 3-mal vergeben) von sechs Städten in den 
1990 er Jahren ${ }^{8}$ (sog. Gesamtkorpora) sowie den bundesweit 30 häufigsten Namen aus dem Jahr 1999 (sog. Frequenzkorpus), die wichtigsten Strukturunterschiede zwischen Mädchen- und Jungennamen synchron untersucht. Das Korpus umfasst insgesamt 446 unterschiedliche Frauenund 405 Männerrufnamen. Dabei ist sie $\mathrm{zu}$ folgenden signifikanten Frgebnissen gelangt (s. Oelkers 2003: 220):

1. Silbenzahl: Frauennamen enthalten durchschnittlich mehr Silben als Männernamen.

2. Hauptakzent: Frauennamen sind durchschnittlich seltener auf der ersten Silbe betont als Männernamen.

3. Konsonanten-/Vokalanteil: Frauennamen enthalten durchschnittlich mehr Vokale, Männernamen mehr Konsonanten.

4. Kernuokal (= betonter Vokal): Frauennamen haben durchschnittlich häufiger einen hellen Kernvokal $(=[e, \varepsilon, i, \mathrm{l}]$, Männernamen seltener

5. Auslaut: Frauennamen lauten durchschnittlich häufiger vokalisch aus, Männernamen häufiger konsonantisch, und Frauennamen lauten durchschnittlich weicher aus, Männernamen härter.

Keine signifikanten Unterschiede gab es bzgl. des Anlauts, der PhonemKeine signifikanten

Unser Korpus basiert, wie erwähnt, auf den bundesweit 20 häufigsten Unser Namen und umfasst insgesamt 560 Rufnamen. Histeresse darin, die Diachronie möglicher struktureller Veränderungen zu erfassen. Dass dafür der Zeitraum von 1945 bis 2008 (mit insgesamt 14 Zeitschnitten) gewählt wurde, ist dem Interesse daran geschuldet, ob tiefgreifende onymische Strukturveränderungen innerhalb und zwischen den Geschlechtern mit solchen sprachexterner Art korrelieren. Damit soll die Hypothese von Gerhards (2003a, b) nochmals überprüft werden. Konkret geht es um folgende Fragestellungen:

1. Sonorität: Auf Basis der realitätsnäheren Transkriptionen sollen mögliche Sonoritätsverschiebungen innerhalb und zwischen den Geschlechtern bzgl. des Auslauts, des Anlauts, aber auch des gesamten Konsonantismus untersucht werden.

2. Anzahl un- bzw. nebcnbetonter Volsale: Es wird zu ermitteln sein, ob die Namen sich bezüglich der Anzahl un- bzw. nebenbetonter Vokale die Namen sich bezüglich der Anzahl un- bzw. nebenbetonter Vokale berücksichtigt werden.

3. Konsonantencluster: Es wird zu ermitteln sein, ob die Namen sich bezüglich der Anzahl an Konsonantenclustern verändert haben.

8 Fs handelt sich um die Städte Bielefeld, Darmstadt, Freiburg, Cottbus, Potsdam un Weimar
4. Hiate: Es wird zu ermitteln sein, ob die Namen sich bezüglich der Anzahl an Hiaten verändert haben.

5. Silbenzahl: Es wird zu ermitteln sein, ob die Namen sich bezüglich ihrer Silbenzahl verändert haben.

6. Akzentstrukturen: Es wird zu ermitteln sein, ob die Namen sich bezüglich ihrer Akzentstrukturen verändert haben.

Natürlich handelt es sich hierbei nicht nur um unabhängige Parameter: Es sind einige Implikationen vorhanden, die sich in den Ergebnissen niederschlagen.

\subsubsection{Sonoritätsberechnungen}

Zu Beginn soll der als stark sexusspezifizierend geltende Auslaut betrachtet werden, genauer die Endsilbe hinsichtlich geschlossen (auf C endend) bzw. offen (auf $\mathrm{V}$ endend). Um diese diachronen Ergebnisse mit Gerhards (2003a, b) und Oelkers (2003) vergleichbar zu machen, wurde auch wenn <er $>[\mathrm{e}]$ (Typ Peter) als offen zu gelten hat - dieser Auslauttyp in Abb. 3 gesondert ausgewiesen, und zwar durch die jeweils schwarz eingefärbten Säulenanteile. Bei den Mädchen spielt der er-Auslaut kaum eine Rolle, bei den Jungen dagegen schon, d.h. die bei Gerhards (2003a: 163) und Oelkers (2003: 185-197) diesbezüglich starke onymische Geschlechterdifferenz nivelliert sich dadurch etwas.

Geschlossene vs. offene Endsilbe

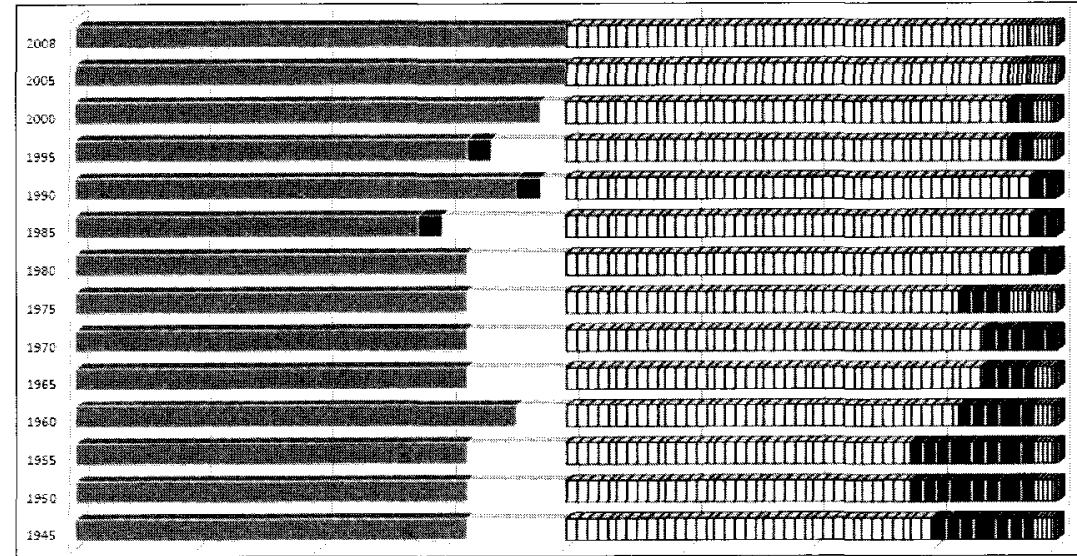

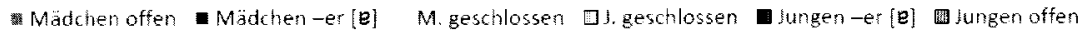

Abb. 3: Geschlossene rs. offene Endsllbe 
Im Zeitverlauf nehmen die männlichen er-Ausgänge stark ab. 2005 und 2008 sind sie gar nicht mehr vorhanden. Es dominiert bei den Jungen klar der geschlossene Namenausgang, bei den Mädchen dagegen der offene, was seit 2005 sogar zu $100 \%$ gilt. Insgesamt sorgt also das Strukturmerkmal offene vs. geschlossene Endsilbe für eine ausgeprägte und persistente onymische Geschlechterdifferenz. ${ }^{9}$

Um die (künstliche) Dichotomie zwischen Vokalen und Konsonanten aufzubrechen, wurden alle Auslaute ihrem Sonoritätswert (gemäß Abb. 2) zugeordnet. Diese Werte wurden pro untersuchtem Jahr addiert und dann durch 20 dividiert. Die Veränderung der Werte nur zwischen 1945 und 2005 zeigt Abb. 4.

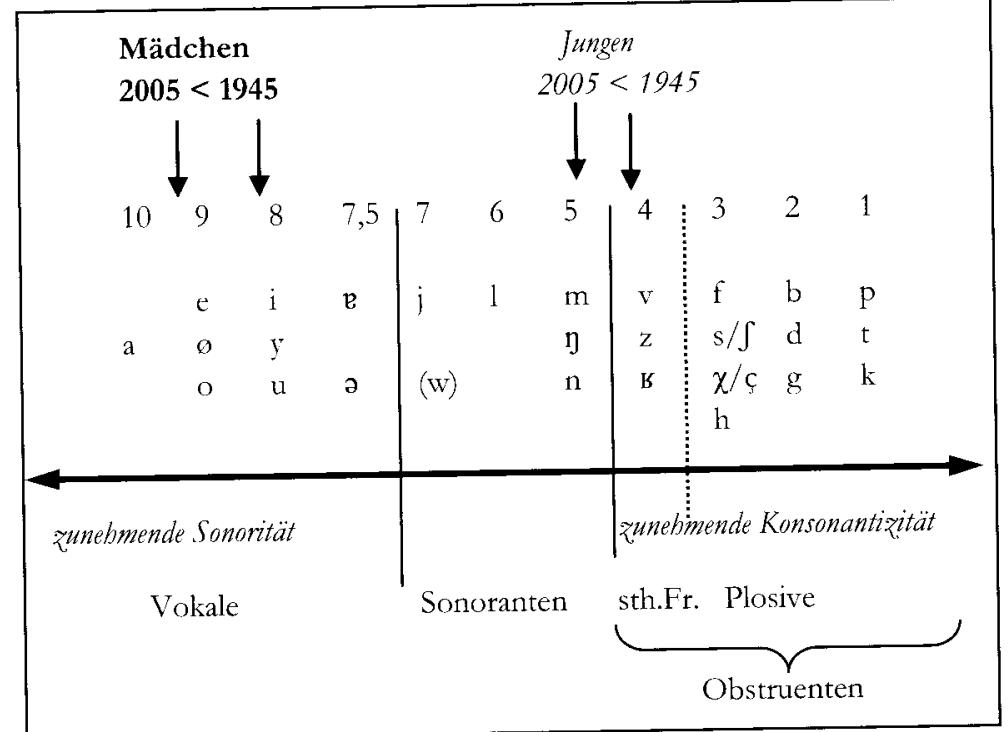

Abb. 4: Auslautsonorität

Beide Geschlechter trennt ein großer Sonoritätsabstand von ca. 4 Sonoritätsgraden. Beide Geschlechter haben außerdem zwischen 1945 und 2005 einen leichten Sonoritätszuwachs erfahren: die Mädchen von 8,35 (1945) auf 9,4 (2005), die Jungen von 4,25 (1945) auf 4,9 (2005). Die

9 Dies bestätigt auch Moser (2009), der sämtliche Namen der Zürcher Neugeborenen von 1988-2008 untersucht. Allein 70\% aller Mädchennamen enden auf -a, weitere 19\% auf -c oder $-n$. Dagegen sind die Auslaute der Jungennamen weitaus diverser: Nur $53 \%$ enden auf -n, -o oder -s (zu Näherem s. Moser 2009: 9). Generell konstatiert Moser bei den Mädchennamen lautliche Monotonie, bei den Jungennamen dagegen weitaus höhere phonologische Varianz. diachrone Differenz beträgt bei den Mädchen 1,05 Sonoritätswerte, bei den Jungen nur 0,65.

Dagegen sind, wie auch schon Oelkers ermittelt hat, die Anlaute zwischen den Geschlechtern ähnlich, obwohl der Anlaut, da meist in der betonten Silbe befindlich, zur salientesten Wortposition zählt. Hier ergaben sich jeweils dicht beieinanderliegende Sonoritätsmittelwerte: Mädchen 4,75 (1945) > 6,25 (2005); Jungen: 3,1 (1945) > 4,45 (2005)

Sie nehmen zwischen 1945 und 2005 bei beiden Geschlechtern leicht zu, nämlich um 1,5 Sonoritätspunkte bei den Mädchen und um 1,35 bei den Jungen.

\begin{tabular}{|l|l|l|l|l|l|l|}
\hline $\begin{array}{l}\text { Kons. } \\
\text { total }\end{array}$ & $\begin{array}{l}\text { Obstru- } \\
\text { enten }\end{array}$ & $\begin{array}{l}\text { Sono- } \\
\text { ranten }\end{array}$ & Jahr & $\begin{array}{l}\text { Sono- } \\
\text { ranten }\end{array}$ & $\begin{array}{l}\text { Obstru- } \\
\text { enten }\end{array}$ & $\begin{array}{l}\text { Kons. } \\
\text { total }\end{array}$ \\
\hline 59 & 40 & 19 & $\mathbf{1 9 4 5}$ & 17 & 39 & 56 \\
\hline 61 & 42 & 19 & $\mathbf{1 9 5 0}$ & 20 & 38 & 58 \\
\hline 55 & 37 & 18 & $\mathbf{1 9 5 5}$ & 21 & 37 & 58 \\
\hline 62 & 42 & 20 & $\mathbf{1 9 6 0}$ & 23 & 39 & 62 \\
\hline 64 & 47 & 17 & $\mathbf{1 9 6 5}$ & 20 & 41 & 61 \\
\hline $\mathbf{5}$ & 42 & 22 & $\mathbf{1 9 7 0}$ & 22 & 41 & 63 \\
\hline 63 & 33 & 30 & $\mathbf{1 9 7 5}$ & 26 & 40 & 66 \\
\hline 58 & 34 & 24 & $\mathbf{1 9 8 0}$ & 26 & 47 & 73 \\
\hline 62 & 37 & 25 & $\mathbf{1 9 8 5}$ & 25 & 47 & 72 \\
\hline 63 & 37 & 26 & $\mathbf{1 9 9 0}$ & 26 & 42 & 68 \\
\hline 49 & 24 & 25 & $\mathbf{1 9 9 5}$ & 31 & 28 & 59 \\
\hline 44 & 16 & 28 & $\mathbf{2 0 0 0}$ & 34 & 25 & 59 \\
\hline 38 & 11 & 27 & $\mathbf{2 0 0 5}$ & 31 & 24 & 55 \\
\hline 37 & 11 & 26 & $\mathbf{2 0 0 8}$ & 28 & 23 & 51 \\
\hline
\end{tabular}

Tab. 2: Sonoranten, Obstruenten und Konsonanten gesamt: absolut

Die größten diachronen Veränderungen haben sich indessen in der Gesamtsonorität des Konsonantismus abgespielt. Hierzu wurde die Gruppe der Sonoranten, zu denen auch der Halbkonsonant /j/ zählt, von den restlichen Konsonanten, d.h. den Obstruenten, getrennt erhoben. Abb. 5 zeigt das Resultat. Den Kurven liegen dabei die Zahlen der Sonoranten aus Tab. 2 zugrunde, die in Prozente umgerechnet wurden.

Deutlich steigen bei beiden Geschlechtern die Sonorantenanteile an, wobei sie gemeinsam bei ca. 30\% im Jahr 1945 starten. Ab 1965 und insbesondere 1990 schießen die Werte in die Höhe, um bei den Mädchen 2005 bei ca. $70 \%$ anzugelangen, bei den Jungen bei $55 \%$. 


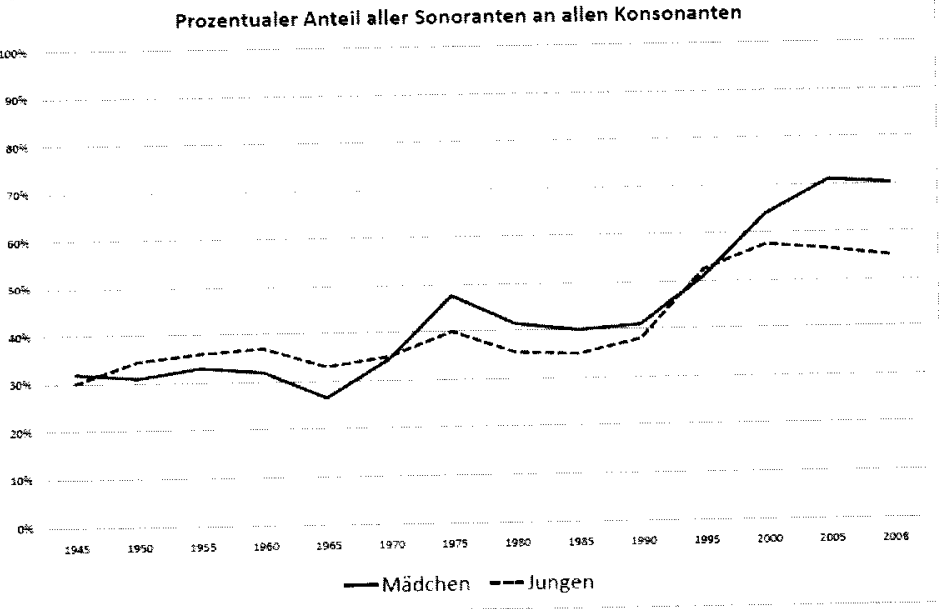

Abb. 5. Prozentualer Anteil allet Sonoranten an allen Konsonanten

Da auch stimmhafte Frikative, d.h. [v, z, b], die Gesamtsonorität erhöhen, wurden sie in Abb. 6 den Sonoranten zugesellt. Hier treiben sie die Werte bei den Mädchen auf $90 \%$, bei den Jungen bleibt es bei den $55 \%$. Typische Namen mit stimmhaften Frikativen sind $\underline{S} u \underline{s} a n n e, \underline{S} a \underline{a} a b, \underline{V}$ anessa, Lisa- Sebastian, S $\underline{\text { ven }}$

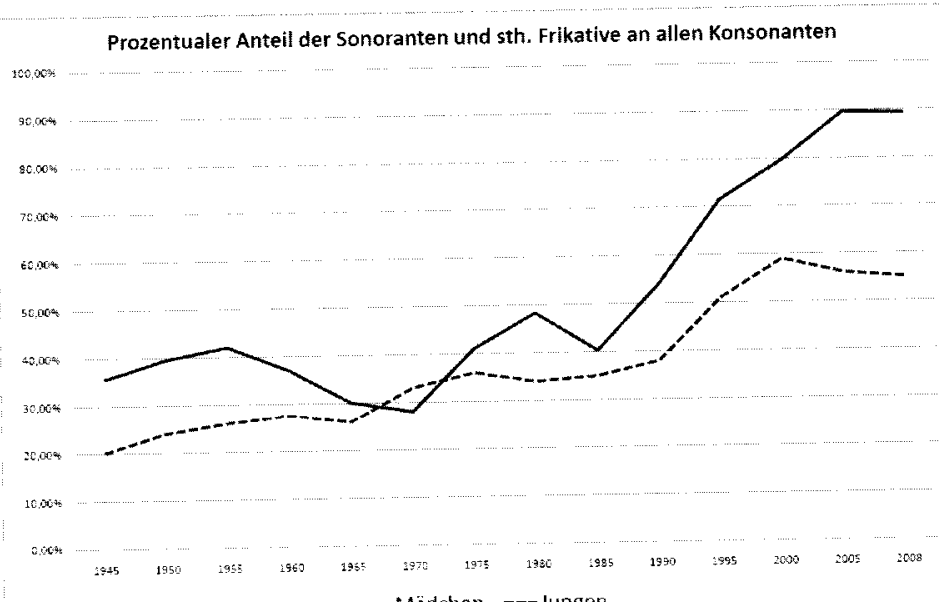

Abb 6. Anteil der Sonoranten und stimmhaften Frikative an allen Konsonanten

Zurück zu den Sonoranten: Bei der Durchsicht der Namen fällt auf, dass es nicht nur der reine Anteil an Sonoranten ist, der zunimmt, sondern dass es vor allem freie, d.h. nicht in konsonantischer Nachbarschaft stehende Sonoranten sind, die vermehrt hinzukommen. So entfaltet das intervokalische [1] in Neele oder das prävokalisch anlautende [1] in Laura mehr Sonorität als konsonantisch gedecktes [1] in Silke oder Claudia. "Gedeckt" bezeichnet hier mindestens einen Konsonanten neben dem Sonoranten, gleich ob er ihm vorangeht oder folgt. Lilli, Platz 12 im Jahr 2008, vereint gleich zwei freie [I] und besteht nur noch in einer Reduplikation, einer Art Lallform. Ähnliches gilt für [j] (und gleichzeitig weitere Sonoranten) bei Maja und Iulia (beide 2008) gegenüber Katja (1975). Auch bei den Jungen spielt sich Ähnliches ab, vgl. Maximilian mit gleich vier freien Sonoranten, ebenso Leon, Elias und Lulian (alle 2008) mit Bernd, Helmut, Klaus (1945). Durch die einerseits abnehmende Namenlänge (s. hierzu Abschnitt 2.3.5) und den gleichzeitig zunehmenden Sonorantengehalt ballt sich maximale Sonorität auf einem minimalen Namenkörper. Da sich dieser Trend heute fortsetzt, seien hier die Namen von 2008 aufgeführt.

\begin{tabular}{|l|l|l|}
\cline { 2 - 3 } \multicolumn{1}{c|}{} & Mädchen & Jungen \\
\hline 1 & Hanna(h) & Leon \\
\hline 2 & Leonie & Lukas \\
\hline 3 & Lea(h) & Luka \\
\hline 4 & Lena & Tim(m) \\
\hline 5 & Mia & Finn \\
\hline 6 & Anna & Luis \\
\hline 7 & Emily & Jonas \\
\hline 8 & Lara & Felix \\
\hline 9 & Laura & Paul \\
\hline 10 & Sara(h) & Maximilian \\
\hline
\end{tabular}

\begin{tabular}{|l|l|l|}
\cline { 2 - 3 } \multicolumn{1}{c|}{} & Mädchen & Jungen \\
\hline 11 & Emma & Max \\
\hline 12 & Lilli & Niclas \\
\hline 13 & Marie & Julian \\
\hline 14 & Lina & Ben \\
\hline 15 & Maja & Elias \\
\hline 16 & Johanna & Jan \\
\hline 17 & Sophie & Noah \\
\hline 18 & Ne(e)le & (Moritz) David (24) \\
\hline 19 & Sofia & Philipp \\
\hline 20 & $\begin{array}{l}\text { (Amelie) } \\
\text { Lisa (21) }\end{array}$ & Jannick/Yannik \\
\hline
\end{tabular}

Tab. 3; Die Top 20 ron $2008^{11}$

Die Tatsache, dass es primär die freien (konsonantisch ungedeckten) Sonoranten sind, die zunehmen, schlägt sich deutlich im Kurvenverlauf von Abb. 7 nieder und gilt für beide Geschlechter, ganz besonders für die Jun gen. Die Sonoranten „befreien“ und entfalten sich also zunehmend im Laufe der Zeit. Die gedeckten Sonoranten stagnieren nicht etwa, sie gehen sogar zurück, insbesondere bei den Jungen.

10 Diese Jiste, die den Berechnungen zugrundeliegt, wurde Ende 2008 ermittelt und nachträglich noch leicht korrigiert: Statt $I$ isa, die faktisch auf Platz 21 gelandet ist, belegt Amelie Platz 20, und statt Daird, der faktisch auf Platz 24 liegt, ist Moritz auf Platz 20 gekommen. Sonst hat sich nichts verändert. Während Darid und Moritz auf exakt den gleichen Gesamtsonoritätswert kommen, ist Amelie etwas sonorer als $L$ isa, verstärkt also die von uns festgestellte Tendenz. 


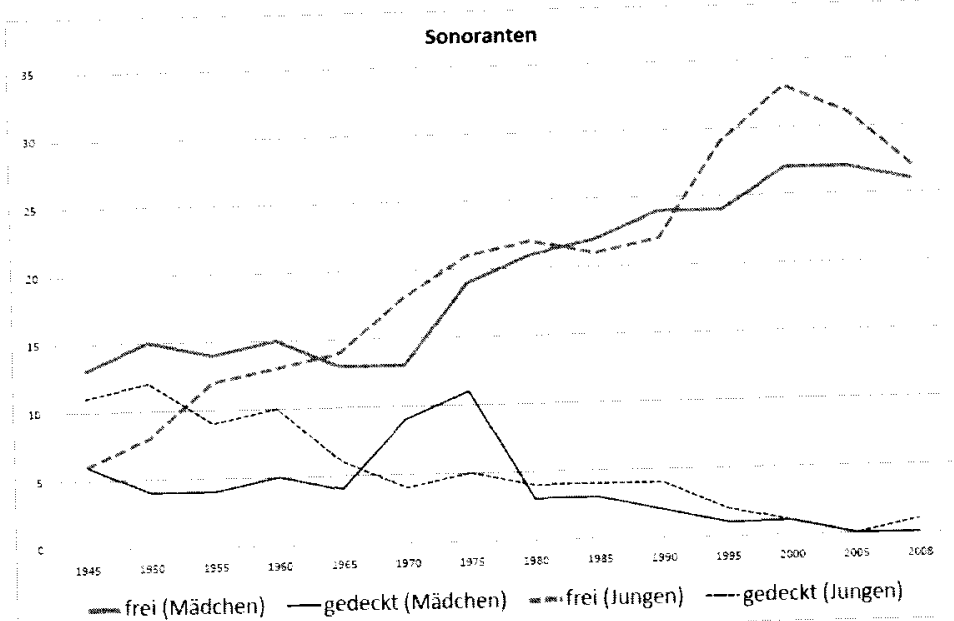

Abb. 7: Freic und (konsonantisch) gedeckte Sonoranten im Vergleich (absolut)

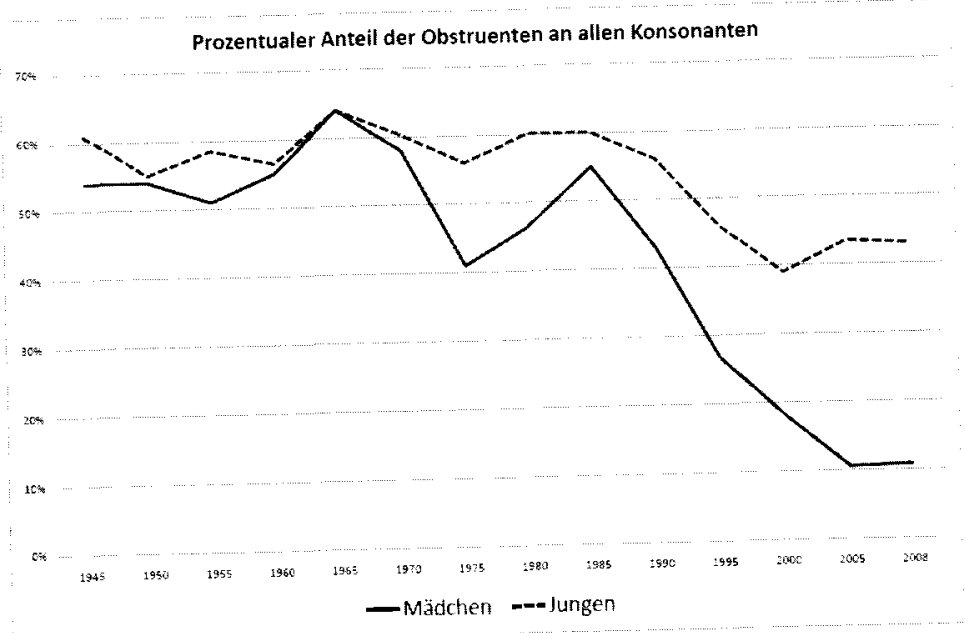

Abb. 8: Anteil der Obstruenten (ohne sth. Frikative) an allen Konsonanten

So verwundert es wenig, dass die Obstruenten sich sukzessive zurückzichen (in Abb. 8 ohne die stimmhaften Frikative, d.h. hier wurden nur die stimmlosen Frikative/Plosive + stimmhaften Plosive berechnet): Bei den Mädchen fallen sie von $54 \%$ auf $10,8 \%$, bei den Jungen von $61 \%$ Bei $43 \%$. Dies lässt den Sonoranten umso mehr Raum und mindert den als ,hart" empfundenen onymischen Gesamteindruck. Moser (2009), der ähnliche Entwicklungen für die Neugeborenennamen des Kantons Zürich beschreibt, spricht hier von „Entschärfung“"

\subsubsection{Un- bzw. nebenbetonter Vokalismus}

Zweifellos tragen mehr als die Konsonanten die Vokale zur Gesamtsonorität eines Wortes bei. Allerdings verringert die zunehmende konsonantische Sonorität die starken Sonoritätskontraste, die bis Anfang der 1970er Jahre die Namen beider Geschlechter prägen (vgl. Brigitte, Heike, Christa bzw. Horst, Dieter, Gerhard, alle 1945). Was den betonten Vokalismus betrifft, so enthält jeder Name mindestens einen Haupttonrokal, d.h. hier kann keine gravierende quantitative Veränderung stattfinden. " Veränderungen vollziehen sich aber bei neben- und unbetonten Vokalen (im Folgenden vereinfachend, unbetonte Vokale“). Abb. 9 dokumentiert den Wandel.

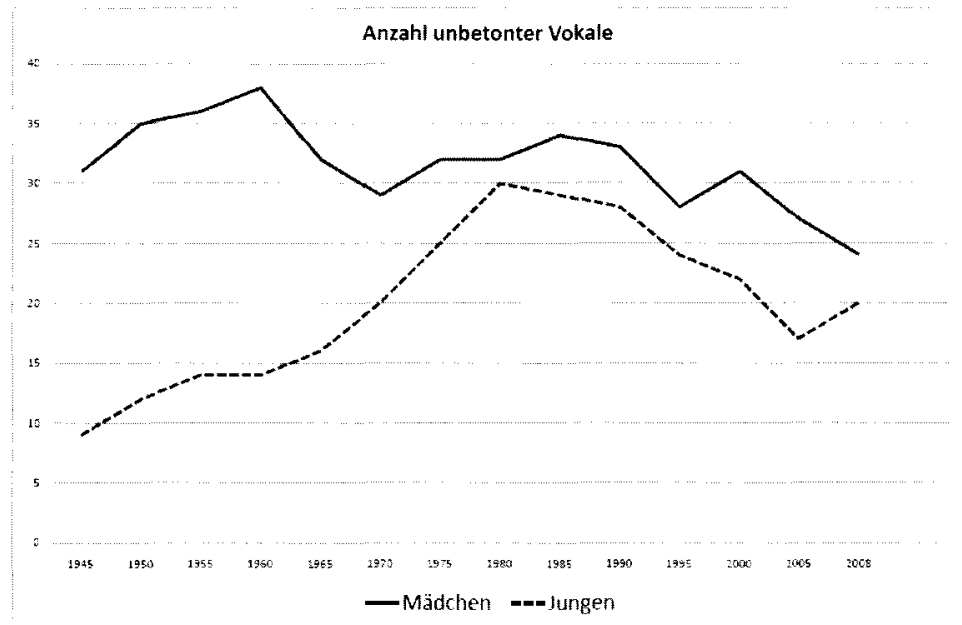

Abb. 9: Veränderungen der Anzahl unbetontcr Vokale (absolut)

Dieser Verlauf legt eine Androgynisierung und damit Gendernivellierung bzgl. dieses Parameters nahe: 1945 starten die Mädchen mit einem mehr als dreimal so hohen Anteil an unbetonten Vokalen (31) wie die Jungen (9). Beide Geschlechter legen bis $1960 \mathrm{zu}$. Die Mädchen verringern seither mit Unterbrechungen ihren Jahresspitzenwert von 38 (1960), während die Jungen stark aufholen und 1980 die Mädchen sogar fast einholen (Mädchen: 32, Jungen: 30). Danach verringern beide Geschlechter mehr oder weniger parallel ihr Aufkommen an unbetonten Vokalen, die Namen

11 Die wenigen germanischen Rufnamenkomposita vom Typ Hannelore, Manfred, Helmut, W'olfgang enthalten zwei betonte Vokale, da aus zwei phonologischen Wörtern bestehend. Dass dabei der erste Bestandreil des Kompositums etwas stärker betont ist als der zweite, entspricht dem deutschen Kompositionsakzent, vgl. Haustür. 
werden kürzer (s. 2.3.5). Trpische Jungennamen 1980 sind Christian, Sebastian, Daniel, Matthias, Andreas, Tobias, Benjamin, Florian, d.h. die zunehmenden Hiate schlagen sehr zu Buche (auch wenn der eine Teil betont ist wie bei Ma'tthias, so bleibt immer auch ein unbetonter Volial, der in diese Rechnung eingeht).

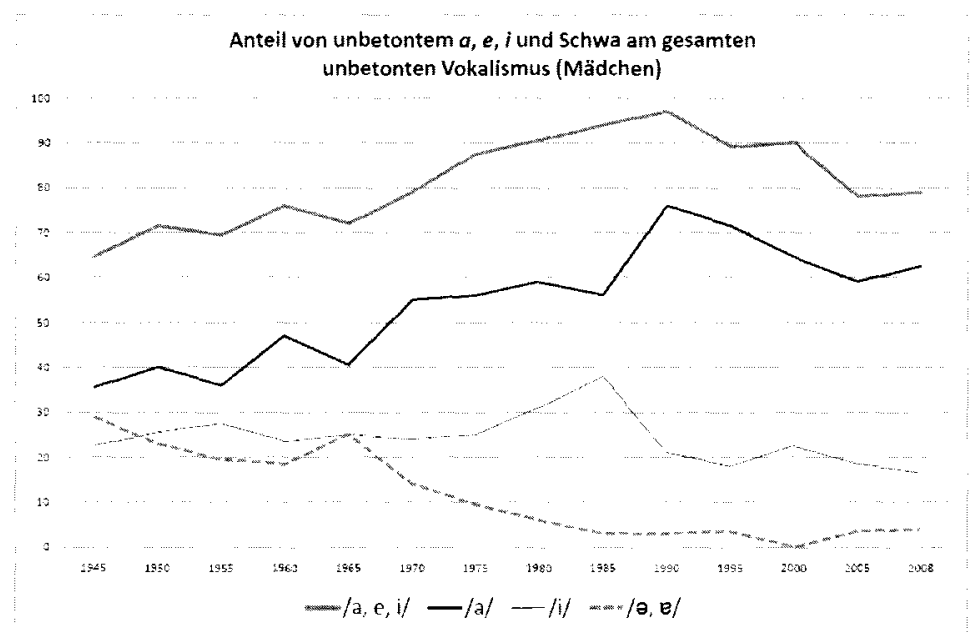

Abb. 10: Antcile von unbetontem [a, e, i] und Schwa (relatir): Mädchen

Schaut man sich die Qualität der Nebentonvokale an, muss man Abb. 10 (Mädchen) mit Abb. 11 (Jungen) vergleichen, denn hier vollziehen sich gerade bei den Jungen wichtige Veränderungen. Der Normalwortschatz (Erbwortschatz) sieht in den Nebentonsilben zum überwiegenden Teil nur Schwa-Laute vor, was seine Ursache in der mhd. Nebensilbenabschwächung hat. Nur einige nebenbetonte Derivationsaffixe erlauben v.a. [I] und [o]: Hündin, kleinlich, Sitzung. Ansonsten kommen volle Vokale nur im Fremd- und Kurzwortschatz vor. Damit nähern sich die Rufnamen strukturell dieser peripheren Lexik an.

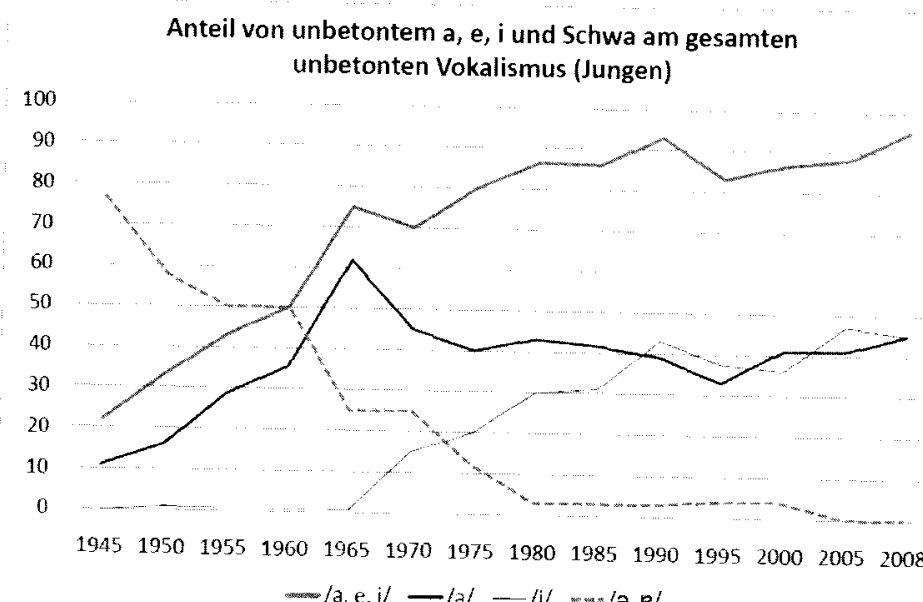

Abb. 11: Anteile von unbetontem [a, e, i] und Schwa (relatir): Jungen

Schwa-baltige Lindsilben standen 1945 bei den Jungen an der Tagesordnung. Typische Repräsentanten sind Günther, Dieter, Peter, Rainer, W'erner, Jürgen, Uwe etc. Andere unbetonte Vokale kamen kaum vor (viele Namen waren ja einsilbig). Dies zeigt deutlich Abb. 11, wo die Schwas bei fast $80 \%$ starten. Bei den Mädchen gab es weniger Schwas, vgl. Brigitte, Hannelore Christel, Heike, Anke. Bei den Jungen herrscht [e] vor, bei den Mädchen dagegen fast nur [ə]. Dies ändert sich in den folgenden Jahrzehnten gründlich, und zwar noch mehr bei den Jungen: Hier schnellen in Abb. 11 die Werte der Vollvokale [a] und [i] nach oben, die oberste Linie verein $[a, e, i]$. Besonders ab 1965 kündigt sich der Durchbruch an: Hier steigen die Werte sprunghaft an, gefolgt von [i], das zeitlich etwas hinterherhinkt und 2005 seinen Höchstwert erreicht, vgl. 'Jannick, Maxírilian, 'Philipp, 'Moritz, 'Felix etc. 1945 existierten solche Namentypen nicht im entferntesten. Ebenso nimmt [a] massiv zu, typische Namen 1965 sind "Thomas, An $n^{\prime} d r e \underline{a}$ s, 'Stefán, 'Michael, Mátttias, 'Christian, 'Olavv. Auch bei den Mädchen finden insgesamt Zuwächse statt, doch enthalten diese Namen schon 1945 zahlreiche volle Nebentonvokale. Die dunklen Vokale [o] und [u] verändern sich wenig im Zeitverlauf. Mit jeweils insgesamt vier unbetonten [o]Silben erreichen die Mädchen 2005 und 2008 ihren Rekord (2008: 'Le.o.nie, So.'phie, So.'fia, Jo.'banna), die Jungen mit drei 1995 (Tó'bi.as, 'Ni.co, 'Le.on). Die absoluten Spitzenwerte für [a] (Mädchen) gelten 1990 mit 25 und für Jungen 1985 mit 13 (von insgesamt 30 Nebentonvokalen; 1965 waren es zwar absolut nur 10 , aber bezogen auf nur 16 Nebentonvokale ergibt sich hier der relative Spitzenwert in Abb. 11). 
7 usammenfassend ist zu sagen, dass sich im unbetonten Vokalismus quantitativ beide Geschlechter stark aufeinander zu bewegen, um sich quantitativ beide Geschlecher sich Jungennamen dann konvergent weitio Mädchennamen an als umgekehrt. Was die Voinsgesamt stärker an die Mädchennamen an als umgekehrt. Was diem sich die kalqualität betrifft, so sind die Verlaufe einseitiger. Hänchennamen an.

Jungennamen noch deutlich stäke Untersuchung der betonten Vokale

Überraschenderweise hat die Unte nichts Spektakuläres erbracht. Die größten Unterschiede und Veränderungen spielen sich ,hinter den Kulissen“ ab.

\subsubsection{Konsonantencluster}

Der Begriff des Konsonantenclusters wird hier weit gefasst: Es handelt sich dabei um jegliche Abfolge mindestens zweier Konsonanten. Es wird sich dabei um wortpositionellen Auftreten unterschieden (Anlaut, nicht zwischen seinem wortpositionellen Auftreten unterschieden (Anlaut, Inlaut, Auslaut), ebenso wenig, ob eine Silbengrenze innerhalb des Clauters verläuft Konsonantencluster konterkarieren in jedem Fall klare Clusters verlaukturen, die von den Rufnamen zunehmend angestrebt werden, in Verbund mit erhöhter Sonorität.

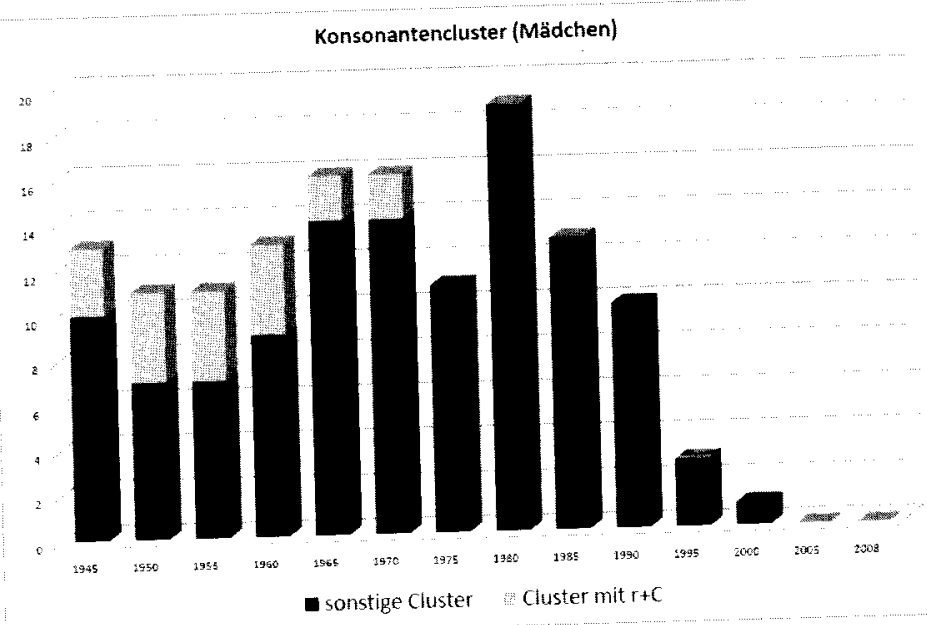

Abb. 12: Vorkommen von Konsonantenclustern (absolut): Mädchen

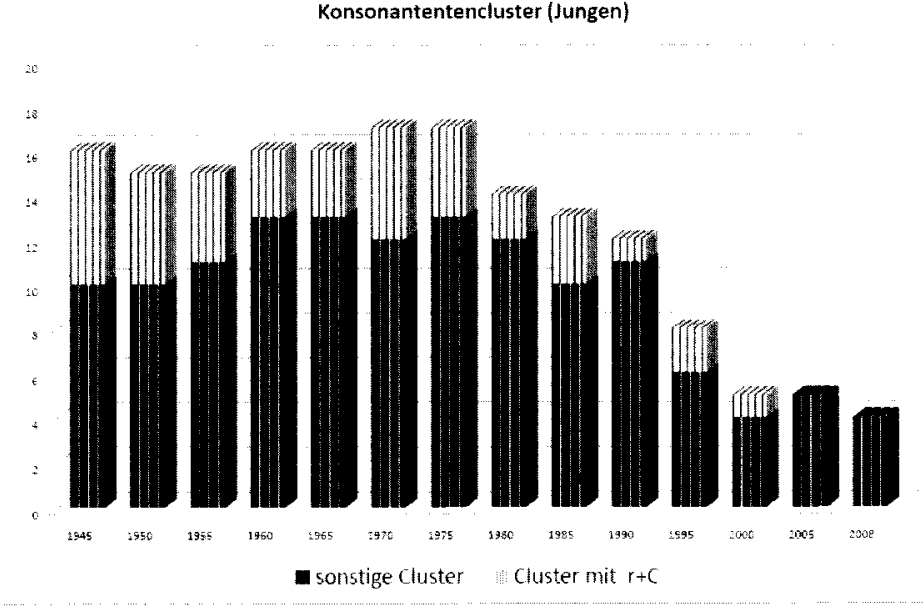

Abb. 13: Vorkommen von Konsonantenclustern (absolut): Jungen

Abb. 12 und 13 enweisen, dass Cluster früher bei beiden Geschlechtern (wenngleich unterschiedlich frequent) vorkamen. Seit 1995 sind jedoch bei beiden Geschlechtern kräftige Einbrüche zu verzeichnen, ganz besonders bei den Mädchen, die seit 2005 nicht mehr einen einzigen Cluster enthalten! Hier herrscht nur noch CVCV. Dies trägt entscheidend zu dem Eindruck bei, dass die heutigen Namen immer weicher würden.

In Abb. 12 und 13 werden die strittigen Verbindungen von $/ \mathrm{r} /+\mathrm{C}$ extra ausgewiesen (die hellen Aufsätze auf den Säulen): Wie bereits gesagt, vokalisiert $/ \mathrm{r} /$ immer vor Konsonant zu [e], weshalb Namen wie Markus, Werner, Gerbard, Ursula, Barbara, Bärbel hier nicht als CC-Verbindungen zu werten sind. Man kann diese grauen Anteile also abziehen, sie werden nur aus traditionellen Gründen berücksichtigt.

\subsubsection{Hiate}

Ähnlich wie volle Nebentonvokale verstoßen auch Hiate gegen die nativen wortphonologischen Regularitäten: Abgesehen von seltenen Fällen wie Bauer, Geier, bei denen der erste Hiatbestandteil in einem Diphthong und der zweite in einem Schwa besteht, sind dem deutschen Erbwortschatz solche Strukturen unbekannt (s. Szczepaniak 2007). Hiate mit zwei Vollvokalen kommen nur in Fremdwörtern vor (Theater, Linguistik). Wie bereits deutlich wurde, stehen die Namen zu Beginn unseres Untersuchungszeitraums den (nativen) Appellativen noch relativ 
nahe. Sukzessive rücken sie strukturell von ihnen ab. Dies wird durch die starke Zunahme an Hiaten unterstützt, was Abb. 14 abbildet.

Hiate

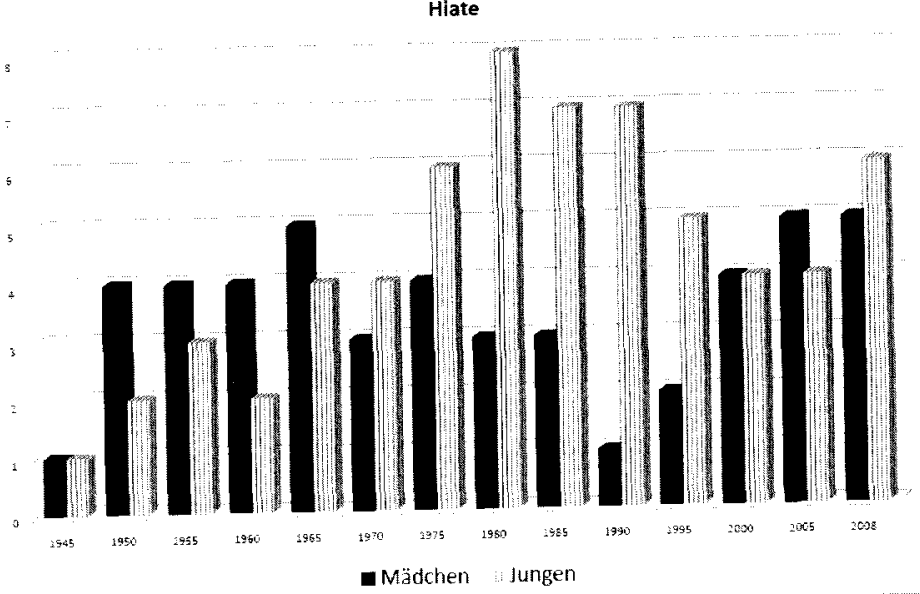

Abb. 14: Die Zunahme an Hiaten bei Mädchen und Jungen (absolut)

Bei den Hiaten wurde weder unterschieden, ob sie im Hauptton vorkomBei den Hialiät sie im Einzelnen besitzen. Wie die men oder nicht noch welche Qualitach machen, sind diese zu gering, als dass man sie weiter ausdifferenzieren sollte. Überraschenderweise haben dass man siaten, insbesonder gerade die Jungennamen einen hohen Anteil an den Haten, insbesondere von 1970-1995. Hiate tragen wesentlich zu Sonoritätssteigerung bei und verunklaren gleichzeitig die Silbengrenzen. Sie führen zu einer diffusen verunklaren gleichzeitig die einzigen hiatushaltigen Namen in 'Marion und 'Micha.el, (wobei der Hiat nie im Hauptton steht), Non diese gerade bei den Jungen bis 1980 in die Höhe: Hier sind so schießen diese gerade bei den Jungen bis 1980 in die tobhe Hier sind es acht (von 20) Jungennamen: 'Christian, Se'bastian, Michael, 'Dame 'Florian, Ma'tthias, An'dreas, To'bias (Mädchen: 'Julia, 'Claudia, Da'niela). Die in als ersten und [a] als zweiten Bestandteil. 2008 sind es meister 'Lea, So'fia, E'milie (als Variante zu Emily) - 'Leon, 'L(o)uis, Maxi'milian, 'Julian, E'lias, 'Noab. Hier sind die (E) vielfätiger als 1980 , und vor allem stehen nun die konkreten Bestandtelle vielfaltiger als 1980 , und vor allem stehen num die Hiate vermehrt im Hauptton, was sie starker exponiert und damit wahrnehmbarer macht. Teilweise bestehen die Namen aus kaum mehr als diesem einen betonten Hiat, d.h. der Hiat wechselt zunehmend von einer Neben- in die Hauptrolle: Mia, Lea, Leon, Lais, Noab.

\subsubsection{Silbenzahl}

Abb. 15 dokumentiert die Veränderungen in der Silbenzahl, die, grob ge sagt, die Namenlänge anzeigt. Dabei ergeben sich naheliegenderweise große Ähnlichkeiten zum Kurvenverlauf ron Abb. 9, die die Anzahl unbetonter Vokale ausweist.

1945 umfasst der weibliche Rufname im Schnitt 2,6 Silben, der männliche nur 1,65, was eine beträchtliche Differenz von fast genau einer ganzen Silbe ergibt. 1960 gelangt der weibliche RufN sogar zu 2,9 Silben, der männliche zu 1,75, d.h. hier steigt die Differen $z$ auf 1,15 Silben an Diese Tendenz bricht danach stark ein. Der minimale Abstand von nur 0,1 Silben stellt sich schon 20 Jahre später im Jahr 1980 ein: 2,6 Silben bei den Mädchen und 2,5 bei den Jungen. Seitdem konvergieren die beiden Kurvenverläufe. Insgesamt wird wieder mehr Kürze angestrebt, was vormals ein typisch männliches onymisches Merkmal war. 2008 beträg die Silbenzahl 2,2 (q) vs. 1,95 (ठ), was eine Differenz von nur 0,25 Silben ergibt. Auf das Gesamtkorpus bezogen umfassen die weiblichen RufN 2,6 und die männlichen 2,0 Silben.

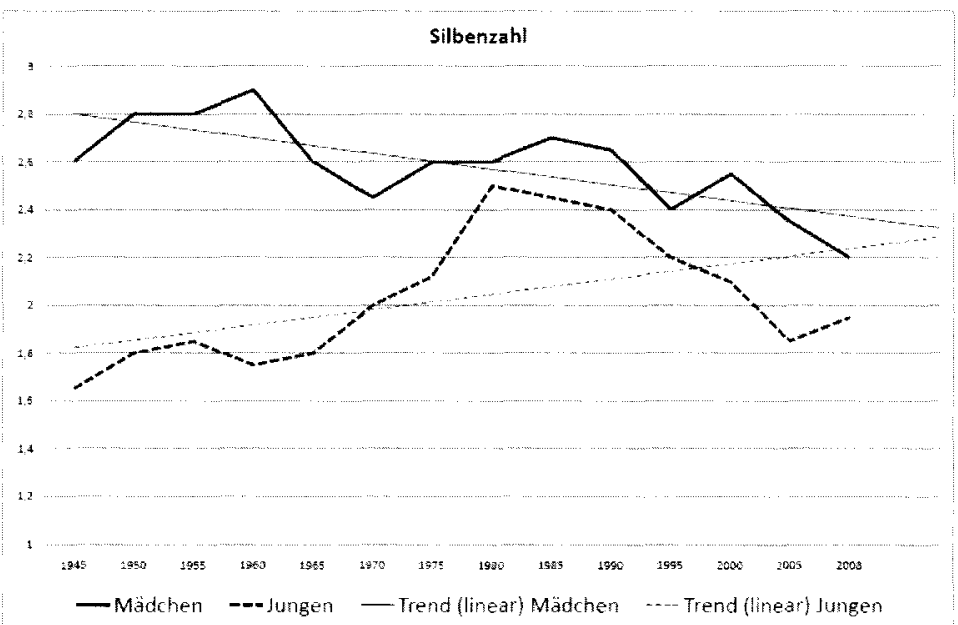

Abb. 15: Veränderungen der Silbenzah

Um zu sehen, wie sich diese Zahlen konkret zusammensetzen, soll die Verteilung der Silbenzahlen nur für 1945, 1975, 2005 und 2008 ausge wiesen werden (s. Tabelle 4): 1945 sind die Mädchennamen in 8 (von 20) Fällen dreisilbig, in weiteren zwei sogar viersilbig, wohingegen die Jungennamen nur einmal dreisilbig sind. Dagegen kommt ihnen exklusiv Einsilbigkeit zu. Diese Extrembesetzungen nivellieren sich im Laufe der 
Zeit. Heute besetzt sogar ein männlicher Fünfsilber die Top 20 (Maximilian)

\begin{tabular}{|c|c|c|c|c|c|c|c|c|}
\hline & \multirow[t]{2}{*}{ Sexus } & \multicolumn{5}{|c|}{ Silbenzahl } & \multirow[t]{2}{*}{ Silben gesamt } & \multirow[t]{2}{*}{ Silben: $\varnothing$} \\
\hline & & 1 & 2 & 3 & 4 & 5 & & \\
\hline \multirow[t]{2}{*}{1945} & 9 & - & 10 & 8 & 2 & - & 52 & 2,6 \\
\hline & $\hat{0}$ & 8 & 11 & 1 & - & - & 33 & 1,65 \\
\hline \multirow[t]{2}{*}{1975} & 9 & - & 10 & 8 & 2 & - & 52 & 2,6 \\
\hline & $\hat{d}$ & 5 & 7 & 6 & 2 & - & 41 & 2,05 \\
\hline \multirow[t]{2}{*}{2005} & 9 & - & 13 & 7 & - & - & 47 & 2,35 \\
\hline & $\hat{0}$ & 7 & 11 & 1 & - & 1 & 41 & 1,6 \\
\hline \multirow[t]{2}{*}{2008} & 9 & - & 16 & 4 & $z$ & - & 44 & 2,2 \\
\hline & $\hat{\lambda}$ & 6 & 11 & 2 & - & 1 & 39 & 1,95 \\
\hline
\end{tabular}

Tab. 4: Die konkiteten Silbenzahlen (Tokens) für 1945, 1974, 2005 und 2008

Ein Blick auf die Namentypes offenbart, dass es fast keine einsilbigen Frauennamen gibt, doch jede Menge bei den Männernamen, d.h. hier verhindert schon das Inventar den Zugriff auf kurze Frauennamen (der einzige in meinem Korpus befindliche ist Kim 1995 auf Platz. 20). Seibicke (1982: 104-106) hat sämtliche Namen (außer den Bindestrichnamen) seines Vornamenbuchs (Seibicke 1977) auf ihre Silbenzahl hin untersucht und kam auf folgende Werte (s. auch Frank 1977):

$\begin{array}{lll} & \text { weiblich } & \text { männlich } \\ \text { einsilbig } & 1 \% & 17 \% \\ \text { zweisilbig } & 40 \% & 37 \% \\ \text { dreisilbig } & 40,5 \% & 38 \% \\ \text { viersilbig } & 17 \% & 7 \% \\ \text { fünfsilbig } & 1,5 \% & 1 \%\end{array}$

Diese Zahlen machen deutlich, dass das Inventar fast keine weiblichen Einsilber vorsieht (Ausnahme: Rutb) und fast keine männlichen Fünfsilber (Ausnahme: Maximilian). Dass Frauennamen im Schnitt länger sind, wird oft durch die Movierung männlicher Namen durch eine Extrasilbe begründet (Typ Martin $\rightarrow$ Martina, man beachte auch den Akzentwechsel). In unserem Material ist dieser Typus nur selten vorhanden (Gabriele, Martina, Manuela, Stephanie) und schlägt damit auch kaum zu Buche. Doch könnte dieses Prinzip das prosodische Schema langer, nicht initialbetonter Frauennamen begünstigt bzw. mitgeformt haben.

\subsubsection{Akzentstrukturen}

Große Unterschiede offenbaren auch die Akzentstrukturen, selbst wenn die Silbenlänge gleich ist, und dies sowohl in diachroner als auch sexusspezifischer Hinsicht. Dabei hat jede Abweichung vom Initialakzent als nichtnative Struktur zu gelten. Bei Abb. 16 und 17 springen eklatante Unterschiede ins Auge. Gleiche Typen wurden jeweils gleich eingefärbt bzw. strukturiert: Einsilber sind weiß 7 weisiber gleich eingefarbt schrafiert = initis schraffiert (dunkel The inalbetont), Drei- und ehrsilber sind schwarz (initialbetont) bzw. grau (nichtinitialbetont).

Einsilbigkeit eignet, wie schon gesagt, ausschließlich Jungennamen. Das ist nicht selbstverständlich, denn die Types (als gesamtes Nameninventar) sagen nichts über die Tokens aus. So wird auch im diachronen Verlauf deutlich, dass sich die Eltern 1980 und 1985 bei ihren diachronen selten für einsilbige Namen entsch 1980 und 1985 bei ihren Sohnen nur Trend wieder zurück.

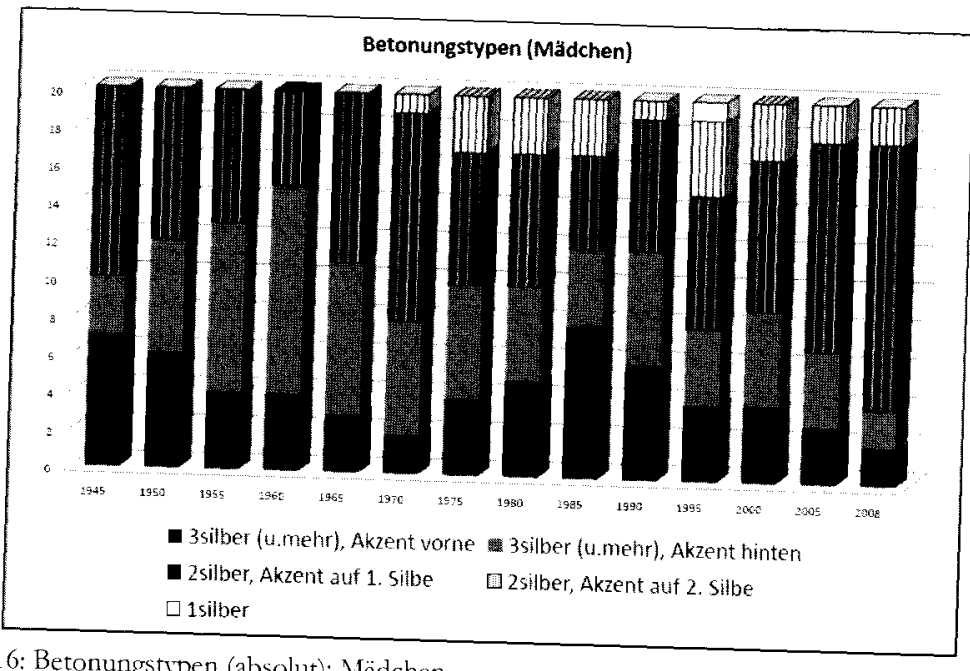

Abb. 16: Betonungstrpen (absolut): Mädchen 


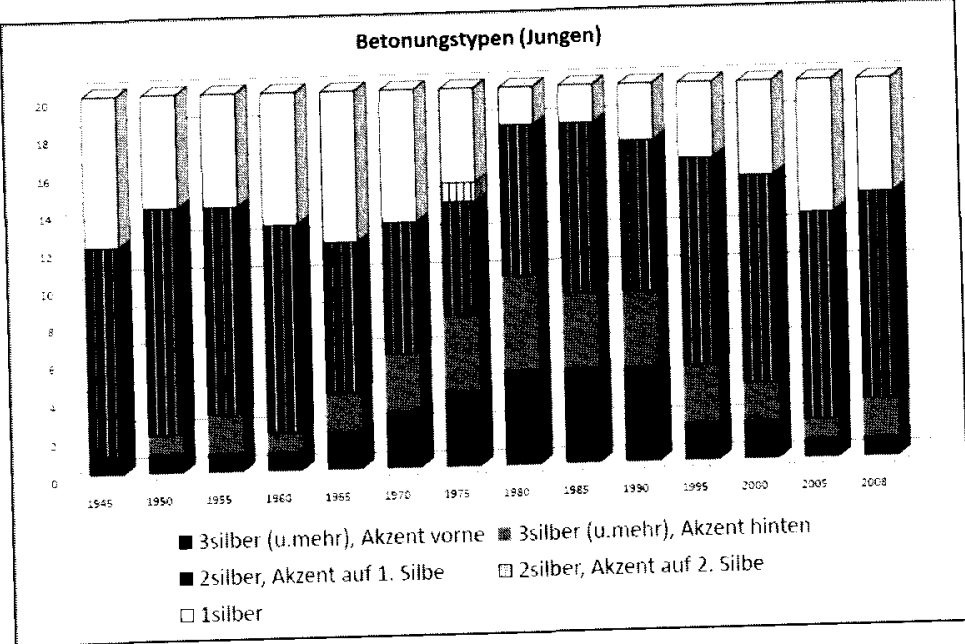

Lbb. 17: Betonungstypen (absolut): Jungen

Der schraffierte Bereich betrifft die Zweisilber: Was für die Mädchen der Der schrafferte Bere Jungen der finalbetonte Zweisilber: Er kommt bei Einsilber, ist fur die Jungen der finalbetonte Zweisiber: Er kommt bei ihnen (so gut wie) nicht vor (1975 gibt es Andre/ Andre, was ein Schwankungsfall ist). Bei den Mädchen ist dieser Typus mit Nadine, Nicole, Yvonne, Michelle, Marie, Sophie durchaus vertreten, wenn auch nicht dominant (s. die llen Schraffuren in Abb. 16). Die initialbetonten Zweisilber stellen bei 等 den Jungen den unmarkierten Typ dar. Ahnliches gilt bei den haben die Große Unterschiede offenbaren die Dreisiber: An diesen haben die Mädchen deutlich größeren Anteil als die Jungen, und sie nutzen dabe Madchen deuttich gerstärkt 1945 und dann wieder 1985 stark die Nichtinitialbetonungen, verstarkt 1945 und dann wiechilber bi Bei den Jungen ist der Verlauf anders: Hier brechen sich die Dreisiber bi 1980 sukzessive Bahn, um in den 1980 er Jahren ihren Höhepunkt zu 1980 sulkzessive Bahn, um in den erreichen. Seit 1995 geht ihr Anteil wieder zurück. Dabei verhalten sich die unterschiedlichen Betonungstypen ähnlich. In jedem Fall nähern sie sich hierin den weiblichen Rufnamen an, auch wenn dieser Parameter insgesamt weiterhin für eine große Geschlechterdifferenz sorgt, ähnlich stark wie der Auslaut.

\section{Lilli, Lina, Mia \& Co: Infantilisierung - Informalisierung -} Intimisierung? 12

In Abschnitt 2.2 wurde erwähnt, dass ein eingetragener Maximilian im Alltag durchaus Max, vielleicht auch Maxi genannt werden kann. Bei den Mädchen kommen Mia und Maja neben Marie sowie Emma neben Emilie vor. Auch wenn in den Top-Positionen desselben Jahrgangs Voll- und Kurzformen nebeneinander stehen, so heißt dies nicht, dass die Vollformen im Alltag auch verwendet werden. Es zeigt aber, dass die Eltern sich als Etikett für ihr Kind für die distanzierendere Originalform entscheiden. Der Eintrag von Kurzformen zeigt dagegen, dass auch die alltagsnäheren und oft hypokoristischen Kurzformen als offizielle, eingetragene Namen fungieren, d.h. etwas überspitzt: Kindliche, zumindest informell-intime, eher intrafamiliär gebrauchte Namenformen werden offiziell und treten damit an die Öffentlichkeit. Somit dringen konzeptionell mündliche Formen zunehmend in konzeptionell schriftliche Kontexte ein.

Die Frage ist, ob es sich hierbei um einen Sprachgebrauchswandel handelt, wie ihn z.B. Linke (2000) in dem Beitrag ,Informalisierung? EntDistanzierung? Familiarisierung? - Sprach (gebrauchs)wandel als Indikator soziokultureller Entwicklungen“ beschreibt. Hier greift sie das Vordringen informell-familiärer Briefanreden in distanzierten Kontexten („Hallo Frau Linke") auf, das „Tschüssen“ als Abschiedsgruß auch bei sich nicht bekannten Menschen sowie die Neigung von Politikern zu stilistischer Deftigkeit. Dies ist direkter Ausdruck bzw. Vollzug des sozialen Prozesses der Informalisierung (Elias 1989) und Intimisierung (Sennett 2004). Die Tatsache, dass gerade Rufnamen als einzige sprachliche Einheit, auf die Menschen frei zugreifen dürfen, sozialen und kulturellen Wandel widerspiegeln, ist sowohl in der Onomastik als auch in der Soziologie Konsens und bildet die zentrale Voraussetzung für die kultursoziologische Untersuchung von Gerhards (2003a). Damit stellt sich die Frage, ob unsere Rufnamen diese Prozesse widerspiegeln, indem Kurz- bzw. noch eher hypokoristische Formen (vor allem erkennbar an diminutiven Techniken) zunehmen.

Schauen wir ins Jahr 1945, so begegnen uns in den Top 20 durchaus Kurz- und Koseformen. Bei den Mädchen sind dies (nach Seibicke 19962007) Karin (schwed. < Katharina), Bärbel (< Barbara), Elke. (als fries. Koseform von Adelheid), Christa $(<$ ChristinalChristiane), Hannelore $<$ Jobanna und Eleonore), Jutta $(<J$ Judith), Heike $(<$ Heinrike), Christel $<$ Christi-

12 Fin ausschlagrebender Grund, mich mit diesem Thema zu befassen, war eine Medizinstudentin namens $L i l l y$, die sehr unter ihrem Namen litt, da sie sich nicht vorstellen konnte, mit einem solch kindlichen Lallnamen in der Wissenschaftswelt zu reüssieren bzw. als Ärztin ernst genommen zu werden. Damals (2005) kam der Name erst in die Toplisten. 
na/Christiane), Anke $(<$ nd. Diminutiv zu Anna), bei den Jungen Hans $(<$ Johonnes), Peter $(<$ Petrus), Klaus $(<$ Nikolaus), Uwe (fries. Lallform evt. aus ( Ulfried), Bernd $(<$ Bernhard), Dieter $(<$ Dietrich), Heinz $(<$ Heinmich), Rainer $<$ Reinhard), Rolf $(<$ Rudolf) und Gerd $(<$ Gerbard). Teilweise handelt es sich, besonders bei den Jungennamen, um bloße Kürzungen bzw. Kontraktionen. Teilweise handelt es sich auch um aus deutschen Dialekten (bzw. dicht verwandten Sprachen) übernommene Kurz- und/oder Diminutivformen, die wahrscheinlich gar nicht als solche empfunden werden (v.a. bei den Mädchen, s. Elke, Antje, Karin). Schließlich kommt es auch zu transparenten Diminutiven (Bärbel, Cbristeð), die sich ausschließlich bei den Maidch finden und feminines (und nicht etwa neutrales) Genus enthalMädchen finden und ferminines (und nicht etw ten. Eine solche Transparenz liegt bei Heinz nicht mehr vor, da die hypokoristische $\approx$-Bildung vor vielen Jahrhunderten stattfand. Hier müsste man eine Skala errichten, die von minimal bis zu maximal nähesprachlichen bzw. hypokoristischen Namen reicht. Reine Kurzformen wie Peter, Han oder Bernd dürften (wenn überhaupt) einen diesbezüglich geringeren Gehalt haben als historische Hypokoristika wie Heinz oder "fremde“ Kurz- und/oder Diminutivformen wie Anke, Heike, und diese wirken wiederum weniger nähesprachlich und verniedlichend im Vergleich zu transparenten Kurz- und Diminutivformen wie Bärbel und Christel. Vermutlich werden Formen wie Peter, Hans oder Bernd sogar als Vollformen wahroenommen, d.h. historische Kurzformigkeit muss heute nicht mehr als solche wahrgenommen und eingeordnet werden, selbst wenn die einstigen Vollformen wie Petrus, Johannes und Bernbard daneben noch vorkommen. ${ }^{13}$

Schauen wir ins Jahr 2008, so sind die Kurzformen nicht unbedingt zahlreicher, aber verfremdeter, da verstärkt aus anderen und jetzt auch nichtgermanischen Sprachen schöpfend (nach Seibicke 1996-2007): Hanna $(<$ Jobanna), Lena $(<$ Helena, Magdalena), Mia, Maja $(<$ Maria), Lara $<$ $(<$ Jobanna), Lena $(<$ Helena, Magdalena), Mia, Maja $(<$ Mlisabetb), Lina $(<$ Carolina), Ne(e)le $(<$ Cornelia); Leon $(<$ Leon(b)ard), Tim $(<$ Timotheus), Max $<$ Maximilian), Niklas (<Nikolaus), Ben $(<$ Benjamin), Jan (evt. auch Jannik

13 Anders war dies noch im $A$ hd., wozu I.offler (1969) eine interessante Untersuchung mi dem Titel „Die Hörigennamen in den älteren St. Galler Urkunden. Versuch einer soziale Differenzierung althochdeutscher Personennamen" vorgelegt hat. In den Urkunden werden 519 Hörigennamen Namen der Unfreien) und 1250 Donatorennamen (Namen der Ereien HerrInnen) erwähnt. Dabei werden die Hörigen mit 33\% (weiblich) bzw. $36^{\circ}$ Freien, Her lutich öfer mit Kurznamenformen bezeichnet als dic Donatoren $(16 \%$ weib(mannlich) deutleh of

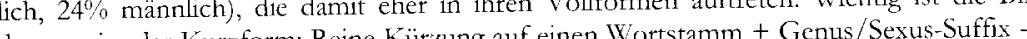
dungsweise der Kurzform: Reine Kurzung auf einen Wortstanm + Genus/Sexus-Suffx bzw. -o dominieren in beiden sozialen Gruppen, mehr noch bei den Do stark hspokorisierende Gemination (Typ Appo) oder Diminuio doch viel häufiger bei den Hörigen vor (zu insgesamt $44^{\circ} \%$ ).
$<$ Jobannes). Hier stehen die Kurzformen im Gegensatz zu 1945 in größerer Distanz zum deutschen phonologischen Wort: Namen wie Bärbel, Christel, Eilke, Anke oder Peter, Uwe entsprechen dem Trochäus mit Reduktionssilbe voll und ganz - im Gegensatz zu solchen Namen wie Mia, Lilli, Emma oder Leon, die zwar trochäisch sind, doch ohne Schwa-Silbe. Diese neuen Namen unterscheiden sich auch kaum bzgl. ihrer Kurz- bzw. Vollformigkeit, d.h. die Kurzform Mia unterscheidet sich nur gering von Vollformen wie I ea oder Noah. Vor allem sticht bei den heutigen Namen, wie in Abschnitt 2 gezeigt, ihre höhere Gesamtsonorität ins Auge, ihre Kürze sowie ihr Reichtum an Hiaten mit Vollvokalen.

Was am ehesten den Eindruck einer gewissen Intimität oder Infantilität vermittelt, ist das, was man „Lallformigkeit" nennen könnte, d.h. bestimmte reduplikative Techniken, die schon kleine Kinder beherrschen. Diese sind bei den Mädchennamen ausgeprägter: Nicht nur sinken, wie gezeigt, die Sonoritätskontraste im Namenwort, sondern die verarbeiteten Vokale und Konsonanten werden sich qualitativ immer ähnlicher: Bei Anna, Hanna und v.a. Lilli springt dies sofort ins Auge, aber auch Neele, Maja, Lara, auch Laura, Lena, Lina, Emma, Jana, Sarab enthalten sehr ähnliche Laute, nämlich [a, i, e] und besonders häufig Dentale. Damit nähern sich die ohnehin wenigen Silben auch noch einander an. Dies ist bei Jungen weniger ausgeprägt: Hier tritt 2008 eher der Typus kurz, aber durchaus mit Sonoritätskontrast zwischen An-, In- und evt. auch Auslaut auf den Plan: Tim, Finn, Ben, Max, Luis, Paul. Diese Einsilber (die nicht alle aus Kürzungen resultieren) tragen noch eine gewisse Kontur, die bei den Mädchennamen nicht mehr besteht. Diese Konturen waren bei den früheren einsilbigen Jungennamen noch ausgeprägter, sowohl bzgl. der Sonoritätskontraste als auch der Konsonantencluster. Letztere kommen heute fast nicht mehr vor, vgl. noch 1945 Hans, Horst, Heinq, Klaus, Rolf, Bernd, Gerd. Die heutigen Einsilber sind weicher/sonorer und entsprechen eher der Struktur CVC (Tim, Ben, Pauh). Extrem viel Sonorität (und Kontrastarmut) kennzeichnet indessen die Jungennamen Leon, Jan und Noab, die sich (besonders Noab) stark den weiblichen Namenstrukturen annähern. Allerdings - und dies unterscheidet sie von den Mädchennamen enthalten sie keine Lallstrukturen und enden sie nicht auf hypokoristisch wirkendes $-i$. Beides setzt zumindest Zweisilbigkeit voraus, und die eignet immer noch mehr den Mädchen- als den Jungennamen.

Für die den deutschen Namen sehr ähnlichen Zürcher Babynamen 1988-2008 konstatiert Moser (2009: 18) eine „Regression zur Kindlichkeit", da die Namen kürzer und v.a. einfacher werden, insbesondere bei den Mädchen. Zum einen verarbeiten sie weniger Laute, zum anderen werden solche Laute onymisch präferiert, die im kindlichen Spracherwerb früh gebildet werde. Die Namen werden ,kindgerechter" (13): 
Möglichenweise ist es am Ende gerade die Vereinfachung und Verkürzung der Namen, ihre Regression zur Kindlichkeit, die den Schlüssel zur Erklärung des Wamen, ihre Rëhält. In einem Zeitalter historisch gesehen außerordentlich niedriger Geburtenraten, in dem die Geburt eines Kindes ein seltenes und meist sorgfältig geplantes Ereignis ist, wird auch das Kind, die Kindheit überhaupt, von den namengebenden Eltern anders wahrgenommen. Die Kindheit hat als Lebensabmengetencen Eigenwert erhalten, den sie früher nicht hatte. Vielleicht sind die schnitt einen Eagentert erhalten, den sie fruher nich hes lautlich einfachen und kurzen Namen Ausdruck eines Zeitalters, in dem Kinder als solche benannt werden - und nicht wie das früher fraglos der Fall war, als zukünftige Frwachsene (Moser 2009: 18).

Eine zunehmende Informalisierung und Intimisierung lässt sich aus unserem Namenmaterial solange nicht ableiten, wie wir nicht wissen, ob Kurzformen wie Gerd, Peter und Uwe nicht ebenso informell wirken wie Tim, Jan oder Niklas. Gerade fremdsprachliche Kurzformen, wie sie heute vermehrt vorkommen, haben den Kürzungsprozess ja nicht im Deutschen vollzogen und transportieren womöglich nicht die entsprechenden Assoziationen.

Was mit Sicherheit gilt, ist, dass hypokoristische Rufnamen mit Diminutivsuffixen oder Lallstrukturen häufiger bei Mädchen als bei Jungen anzutreffen sind, d.h. möglicherweise tut sich hier eher eine konstante Geschlechterdifferenz auf (Mädchennamen erfahren generell eine stärkere Infantilisierung und Intimisierung) als eine gesellschaftliche Gesamtentwicklung. ${ }^{14}$ Vergleicht man die heutigen Namen mit denen vor 60 Jahren, so stellt sich die Frage, wie man echte Diminutiva wie Christel und Bärbel so stellt sich die Frage, wie mal me mit heutigen Lallnamen wie Lilli/Lilly, Lina und Neele verrechnet. Auch müsste man die Top-20-Gruppe verlassen und viel tiefer in das Namenmaterial eindringen. Schon ein Blick auf die jeweils ersten 50 Rufnamen von 2008 offenbart hier interessante Unterschiede: Bei den Mädchen wird der schlicht-sonore Klangtypus mit Lisa, Leni, Lucy, Jule, Pia, Amy, Nina eher fortgesetzt als bei den Jungen, bei denen sich andere, profiliertere Namenstrukturen auftun. Hier eröffnet sich ein großes und vielversprechendes Forschungsgebiet.

\section{Fazit}

Die Frage, ob zwischen 1945 und 2008 eine Androgynisierung der Rufnamenstrukturen stattgefunden hat, ist mit Ja zu beantworten: Noch nie seit 1945 waren sich die Rufnamen beider Geschlechter strukturell so ähnlich wie heute. Damit hat eine onymische Abschwächung von Gender

14 Dics kongruiert mit dem, was Debus (1988) für Kiel beschreibt: Mädchen erhalten, bezogen auf eine Untersuchung im Jahr 1972, 1,5mal häufiger Kosenamen als Jungen. stattgefunden. Die Frage ist, was diese Ähnlichkeit konstituiert und wie sie zustande kommt: Nähert sich eher das eine Geschlecht onvmisch dem anderen an, bewegen sich beide aufeinander zu, vollziehen sie gemeinsam neue Entwicklungen? Wann lassen sich in diesem Zeitraum gravierende Veräncerungen bzw. Umbrüche feststellen?

Divergenzverstärkungen zwischen den Geschlechtern waren nicht feststellbar. Bereits anfänglich vorhandene Differenzen blieben zwar teilweise erhalten (Auslaut, Akzentstrukturen), doch sind für die Mehrzahl der untersuchten Parameter eher Konvergenzentwicklungen feststellbar. Diese Bewegungen verlaufen nicht svmmetrisch. Dabei haben sich etwas öfter die Jungennamen den Mädchennamen angenähert als umgekehrt. Hier ein Resümee der wichtigsten Entwicklungen:

1. Bezüglich der Auslautsonorität besteht im gesamten Zeitverlauf eine deutliche Sonoritätsdifferenz von ca. vier Sonoritätswerten. Im Laufe der Zeit haben die Namen beider Geschlechter etwas an Sonorität gewonnen. Wenn man nur die Opposition offene vs. geschlossene Endsilbe betrachtet, dominiert bei den Mädchennamen eindeutig der offene Ausgang, heute sogar zu 100\%, während die Jungennamen fast ebenso eindeutig die geschlossene Endsilbe präferieren (von aktuellen Ausnahmen wie Luka, Noab abgesehen).

2. Gemeinsam haben die Namen beider Geschlechter stark an Sonorität zugelegt. Am meisten betrifft dies die freien (konsonantisch ungedeckten) Sonoranten und stimmhaften Frikative. Hierin überflügeln v.a. seit den 1990er Jahren die Mädchen- die Jungennamen. Die Anzahl der gedeckten Sonoranten geht zurück. Ob Sonorität mit „Weichheit“", „Sanftheit“, „Lieblichkeit“, „Ungefährlichkeit“, „Schönheit“ etc. assozilert wird und dies wiederum mit Weiblichkeit (Phonosemantik), sei dahingestellt und damit nicht in Zweifel gezogen.

3. Die als ,hart" geltenden Obstruenten (hier die stimmlosen Frikative + alle Plosive) zeigen einen dramatischen Rückgang. Sie starten bei ähnlich hohen Werten 1945 und sinken ab 1970 stark ab, mehr noch bei den Mädchen als bei den Jungen.

4. Bezüglich der Anzahl un- sowie nebenbetonter Vokale starten die Geschlechter auf unterschiedlichen Positionen: Die Mädchennamen enthalten 1945 davon etwa dreimal so viele wie die Jungennamen. Die Jungennamen legen sukzessive stark zu. Die Mädchennamen verringern ihren Anteil leicht, v.a. 1970 sacken ihre Werte ab. 1980 entsprechen sich die Zahlen fast vollständig, um danach gemeinsam leicht abzufallen. Im ganzen Zeitverlauf liegen die unbetonten Vokale bei den Mädchen über denen der Jungen, doch mit deutlich geringeren Differenzen als im Zeitraum von 1945 bis 1960, wo die Werte besonders stark divergieren. 
5. Bezüglich der Qualität un- sowie nebenbetonter Vokale kann man fast nur von einer einseitigen Annäherung der männlichen an die weiblichen Rufnamen sprechen. Besonders bei [a] und [i] legen die Jungennamen zu, umgekehrt rücken sie radikal von den Schwa-Lauten ab. Die Mädchennamen enthielten schon immer mehr [a], [e] und [i] und legen dabei noch zu.

6. Bezüglich der Anzahl an Konsonantenclustern haben beide Ge schlechter starke Rückgänge zu verzeichnen. Seit 2005 kommen sie bei den Mädchen gar nicht mehr vor, die Jungen erreichen die niedrigsten Werte denn je.

7. Bei den Hiaten starten beide Geschlechter bei jeweils nur einem einzigen von 20 Namen. Dies ändert sich bei den Mädchen schnell, die Jungen ziehen nach - und schließlich deutlich an den Mädchen vorbei: Ab 1975 haben Hiate hier Hochkonjunktur, bei den Mädchen gehen sie eher zurück. Heute ähneln sich die Geschlechter wieder. Die Hiate rücken insgesamt unter den Hauptton, d.h. sie wechseln von der Neben- in die Hauptrolle, und dies bei gleichzeitig abnehmender $\mathrm{Na}$ menlänge.

8. Die Silbenzahlverläufe ähneln erwartbarerweise stark den Nebentonvokalverläufen: Große Divergenzen zu Anfang, starke beidseitige Konvergenz bis 1980 und seitdem parallel eine sukzessive Abnahme an Silben, also an Namenmasse. Die anfängliche Differenz von einer Silbe (1945) schrumpft auf heute $1 / 4$ Silbe. Dabei sind die Mädchennamen von 1945 bis heute etwas kürzer geworden als die Jungennamen länger.

9. Bei den Betonungstypen ergeben sich zwar geschlechterintern diachrone Veränderungen, doch ohne gegenseitige Annäherungen. Hier scheint Geschlecht fest und dauerhaft kodiert zu werden. Einsilber waren und sind genuin männlich (und dort auch häufig), finalbetonte Zweisilber genuin weiblich (doch nicht so häufig). Der initialbetonte Zweisilber nimmt bei den Mädchennamen diachron zu und nähert sich hierin eher (den diesen Typus schon immer präferierenden) Jungennamen an. Umgekehrt nimmt Mehrsilbigkeit bei den Jungennamen, zumindest temporär (v.a. 1980), deutlich zu, auch verbunden mit Nichtinitialakzentstrukturen, um heute wieder in die Peripherie abzuwandern.

10. Beide Geschlechter erfahren einen Zuwachs an Gesamtsonorität, eine Abnahme an Sonoritätskontrasten, und, v.a. bei den Mädchennamen, eine Zunahme an Kürze und an „Lallnamigkeit“.

Die größten strukturellen Geschlechterdifferenzen spielen sich also im Auslaut und in der Akzentstruktur ab, kaum im Anlaut und im betonten Vokalismus. Die deutlichsten diachronen Veränderungen fanden im unbetonten Vokalismus, im Konsonantismus (mehr Sonorität, Rückgang an Clustern) und in der Namenlänge (Silbenzahl) statt. Betrachtet man anhand der verschiedenen Kurvenrerläufe die wichtigsten Umbrüche, so stechen die 1970er und auch die 1990er Jahrs ins Auge. Es liegt nahe, dies durch die großen gesellschaftlichen Umbrüchen zu motivieren (1968, zunächst Emanzipation und Gleichberechtigung der Frau, später ihre faktisch voranschreitende Gleichstellung). Was möglicherweise überrascht, ist die Tatsache, dass die männlichen Rufnamen insgesamt mehr Dynamik bewiesen und einen weiteren Weg zurückgelegt haben als die weiblichen.

Der sich immer wieder aufdrängende Eindruck einer sich onymisch manifestierenden Infantilisierung, Informalisierung und Intimisierung wurde nur als Frage formuliert: Kurz- und Diminutivformen, die dies belegen könnten, hat es schon immer gegeben, besonders bei den Mädchennamen. Bevor das Verhältnis zwischen Kurzformen und Nähesprachlichkeit bzw. Familiarität/Intimität nicht genau untersucht ist, lassen sich dazu keine präzisen Aussagen machen. Anders ist dies bei hypokoristischen Strukturen wie Diminutiven und bei monotonen Lautstrukturen in den Lallnamen (Lilli, Nanna, Nina, Nele), wovon in dem hier untersuchten Zeitraum Mädchennamen stärker betroffen sind.

Diese Untersuchung hat sich nur auf die Spitze des onymischen Eisbergs bezogen. Was sich jenseits der Top 20 abspielt, bedarf noch der Erforschung.

\section{Literatur}

Andersson, Thorsten (1998): Germanskt personnamnsskick i indoeuropeiskt perspektiv. In: Andersson, Thorsten u.a. (Hrsg.): Personnamn och social identitet, Stockholm: Kungl. Vitterhetsakademien, 13-35.

Bardesono, Anne (2008): „Naming Gender“ kontrastiv. Phonosemantische Untersuchung zu männlichen und weiblichen Rufnamen im Deutschen und Italienischen. Magisterarbeit, Universität Mainz.

Barry, Herbert/Harper, Aylene (1982): Evolution of Lnisex Names. In: American Name Society, 30/1, 15-22.

Barry, Herberl/Harper, Aylene (1993): Feminization of Unisex Names from 1960 to 1990. In: Names, 41/1, 228-238

Brylla, Eva (2001a): Personnamn och genus. In: Studia anthroponymica Scandinavica, 19, 11-29.

Brylla, Eva (2001b): Personnamn och genus. Manligt och kvinnligt i namnen. In: HarlingrKranck, Gunilla (Hrsg.): Namn i en föränderlig värld, Helsingfors: Svenska litteratursällskapet i Finland, 36-44

Debus, Friedhelm (1974): Namengebung. Möglichkeiten zur Erforschung ihrer Hintergründe. In: Onoma, 18, 456-469. 
Debus, Friedhelm (1976a): Zu Namengebung und Namenverwendung in Nittelalter und Neuzeit. In: Debus, liriedhelm/Hartig, Joachim (Hrsg.): Festschrift für Gerhard Cordes zum 65. Geburtstag Bd. II. Sprachwissenschaft, Neumünster: Wachholtz, $56-67$.

Debus, Friedhelm (1976b): Deutsche Namengebung im Wandel. Dargestellt am Beispiel Schleswig-Holsteins. In: Beiträge zur Namenforschung, 11, 388-410.

Debus, Friedhelm (1977): Soziale Veränderungren und Sprachwandel. Moden im Gebrauch von Personennamen. In: Moser, Hugo (Hrsg.): Sprachwandel und Sprachgeschichtsschreibung, Düsseldorf: Schwann, 167-204.

Debus, Friedhelm (1985): Zur Pragmatik von Namengebung und Namengcbrauch in unserer Zeit. In: Beiträge zur Namenforschung, 29, 305-343.

Debus, Friedhelm (1987): Personennamengebung der Gegenwart im historischen Vergleich. In: Zeitschrift für I iteraturwissenschaft und Linguistik, 45/67, 52-73.

Debus, Friedhelm (1988): Original und Variation. Zur Kreativität bei der Benennung ron Personen. In: Haider Munske, Horst u.a. (Hrsg.): Deutscher Wortschatz. Lexikologische Studien, Berlin/New York: de Gruyter, 24-45.

„Die beliebtesten Vornamen von 1890 bis heute." http://www.beliebte-vornamen.de (Stand: 11.11.2009)

Elias, Norbert (1989): Zivilisation und Informalisierung. In: Elias, Norbert (Hrsg.): Studien über die Deutschen. Machtkämpfe und Mabitusentwicklung im 19. und 20. Jahrhundert, Frankfurt/Main: Suhrkamp, 31-158.

Frank, Rainer (1977): Zur Frage einer schichtenspezifischen Personennamengebung. Namenkundliche Sammlung Analyse und Motivuntersuchung über den Kreis und die Stadt Segeberg, Neumünster: Wachholtz.

Gerhards, Jürgen (2003a): Die Moderne und ihre Vornamen, Wiesbaden: Westdeutscher Verlag.

Gerhards, Jürgen (2003b): Geschlechtsklassifikation durch Vornamen und Geschlechtsrollen im Wandel. In: Berliner fournal für Soziologie, 13, 59-76

Huschka, Denis/Gerhards, Jürgen/Wagner, Gert (2005): Naming Differences in Divided Germany. Research Notes 8. Deutsches Wirtschaftsinstitut Berlin.

Kunze, Konrad (2004): drv-Atlas Namenkunde. Vor- und Familiennamen im deutschen Sprachgebiet, München: dtv.

Lieberson, Stanley/Mikelson, Kelly (1985): Distinctive African American names. An experimental, historical, and linguistic analysis of innovation. In: American Sociological Review, 60/1, 928-946.

Linke, Angelika (2000): Informalisierung? Ent-Distanzierung? Familiarisierung? Sprach(gebrauchs)wandel als Indikator soziokultureller Entwicklungen. In: Der Deutschunterricht, $2,66-77$.

Löffler, Heinrich (1969): Die Hörigennamen in den älteren St. Galler Lrkunden. Versuch einer sozialen Differenzierung althochdeutscher Personennamen. In: Beiträge zur Namenforschung, 4, 192-211.

Moser, Peter (2009): Vornamen klingen heute anders als früher. Entwicklungstendenzen bei der Vornamenwahl von Zürcher Eltern 1988-2008. In: Statistik info, 08/09, 120. Statistisches Amt des Kantons Zürich.

Nübling, Damaris (2009a): Von Monika zu Mia, von Norbert zu Noah. Zur Androgynisierung der Rufnamen seit 1945 aus prosodisch-phonologischer Perspektive. In: Beiträge zur Namenforschung, 44, 67-110.
Nubling, Damaris (2009b): Von Horst und Heloa zu Leon und I eonie. Werden die Rufnamen immer androgtner? In: Der Deutschunterricht, 5, 77-83.

Oelkers, Susanne (2003): Naming Gender. Empirische L'ntersuchungen zur phonologischen Struktur von Vornamen im Deutschen, Frankfurt: Peter Lang.

Oelkers, Susanne (2004): Der Fall Luca. Zur Männlichkeit und Weiblichkeit ron Vornamen. In: Wyss, Exa L. (Hrsg.): „What's in a name?" Namen in sozialen und kulturellen Kontexten. Bulletin VALS/ASL $\Lambda$, 80, 155-170.

Sennett, Richard (2004): Verfall und Ende des offentichen I ebens. Die Trrannei der Intimität, Frankfurt: Fischer.

Seibicke, Wilfried (1982): Die Personennamen im Deutschen, Berlin/New York: de Gruyter.

Seibicke, Wilfried (1977): Vornamen, Wiesbaden: Verlag für deutsche Sprache.

Seibicke, Wilfried (1991): Vornamen, Frankfurt: Verlag für Standesamtwesen.

Scibicke, Wilfried (1994): ,Pränomen ist auch Omen'. Moden in der Vomamengebung in Ost und West. In: Sprachreport, 1, 9-11

Seibicke, Wilfried (1996-2007): Historisches Deutsches Vornamenbuch. 5 Bände, Berlin/New York: de Gruster.

Szczepaniak, Renata (2007): Der phonologisch-typologische Wandel des Deutschen von iner Silben- zu einer Wortsprache, Berlin/New York: de Grutter. 


\section{Linguistik -}

\section{Impulse \& Tendenzen}

Herausgegeben von

Susanne Günthner

Klaus-Peter Konerding

Wolf-Andreas Liebert

Thorsten Roelcke

45

De Gruyter

\section{Genderlinguistik}

Sprachliche Konstruktionen

von Geschlechtsidentität

Herausgegeben von

Susanne Günthner

Dagmar Hüpper

Constanze Spieß 
ISBN 978-3-11-027287-1

e-ISBN 978-3-11-027290-1

ISSN 1612-8702

Library of Congress Cataloging-in-Publication Data

A CIP catalog record for this book has been applied for at the Library of Congress.

Bibliografische Information der Deutschen Nationalbibliothek

Die Deutsche Nationalbibliothek verzcichnet diese Publikation in der Deutschen Nationalbibliografie; detaillierte bibliografische Daten sind im Internet über http://dnb.dnb.de abrufbar.

(C) 2012 Walter de Gruyter GmbH \& Co. KG, Berlin/Boston

Einbandabbildung: Marcus Lindström/istockphoto

Druck: Huhert \& Co. GmbH \& Co. KG, Görtingen

$x$ Gedruckt auf säurefreiem Papier

Printed in Germany

www.degruyter.com

\section{Vorwort und Danksagung}

Der vorliegende Sammelband basiert auf einer interdisziplinären und internationalen Tagung, die unter dem Titel „Sprachliche Konstruktionen ron Geschlechtsidentität" im Norember 2008 an der Westfälischen Wilhelms-Universität der WWU durchgeführt wurde. Die Tagung trug dazu bei, dass namhafte Vertreter der linguistischen Genderforschung, NachwuchswissenschaftlerInnen der Linguistik und Vertreter anderer Fachdisziplinen aktuelle Fragen der Genderforschung diskutierten. Dabei wurden gängige Konzepte kritisch rezipiert, reflektiert und mit neuen Methoden und Zugängen konfrontiert.

Der vorliegende Sammelband kann zwar keine vollständige Zusammenschau der kontroversen und fachübergreifenden Diskussionen im Rahmen der Tagung präsentieren, dennoch kann er mit den versammelten Beiträgen dazu beitragen, eine Basis für weitere anregende und fundierte Debatten im Bereich der Genderlinguistik liefern. Mit den hier versammelten Beiträgen aus der Sprachwissenschaft sowie aus angrenzenden Disziplinen erhoffen wir, einen Einblick in die Aktualität und Vielfältigkeit der Genderlinguistik zu liefern, der zu weiterer Forschung animiert.

Bei dieser Gelegenheit möchten wir uns bei all denjenigen bedanken, die zur Entstehung und dem Gelingen der Tagung sowie der Fertigstellung des Sammelbandes beigetragen haben: Hierzu gehören neben den ReferentInnen/AutorInnen vor allem auch die beteiligten Hilfskräfte und MitarbeiterInnen: Anna-Maria Balbach, Maria Baumeister, Elisa Franz, Sarah Horstkamp und Katharina König. Vera Beckmann und Marcel Fladrich danken wir für die Hilfe bei der Erstellung der Druckvorlagen, Christian Loos für die Mitarbeit am Sachregister.

Ferner gilt unser großer Dank der Gleichstellungsbeauftragten und dem International Office der WWU für ihre finanzielle Unterstützung bei der Tagung wie auch bei der Fertigstellung des Bandes.

Den Reihenherausgebern Klaus-Michael Konerding, Wolf-Andreas Liebert und Thorsten Roelcke danken wir für wichtige Kommentare zu den Manuskripten, dem de Gruyter Verlag für die Aufnahme des Bandes in die Reihe.

Münster, im Sommer 2011

Susanne Günthner, Dagmar Hüpper und Constanze Spieß 